
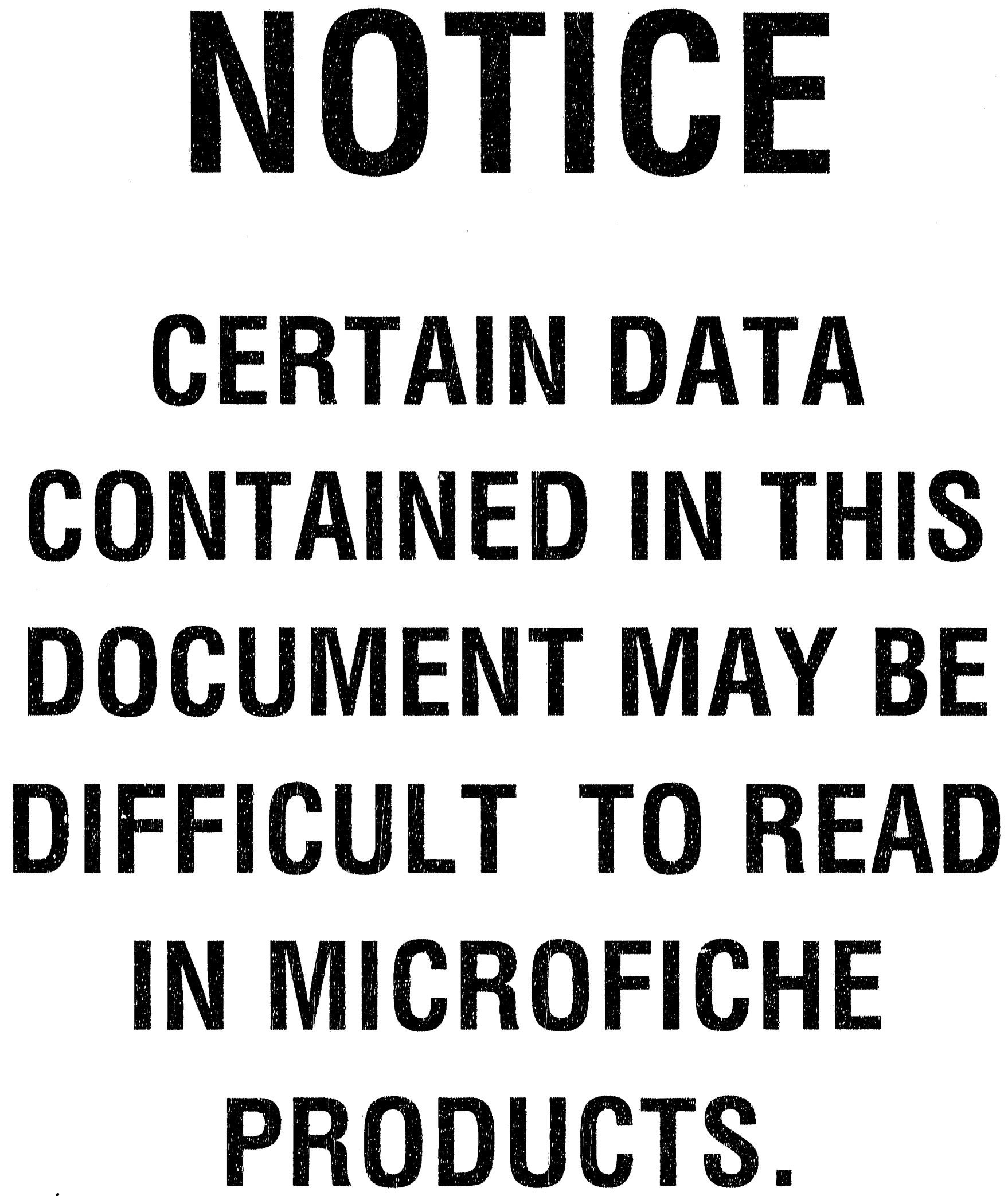


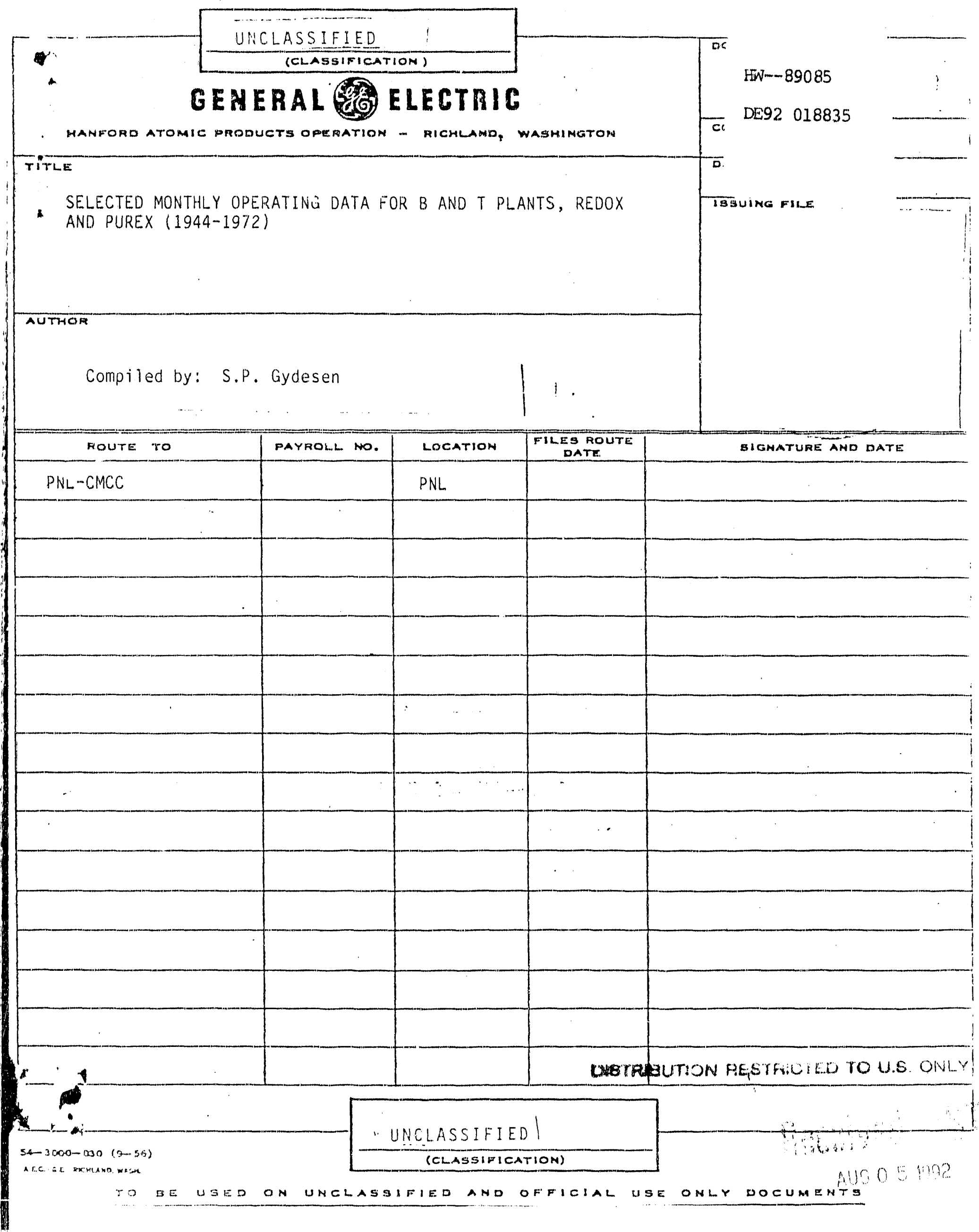


This report was prepared as an account of work sponsored by an agency of the United States Government. Neither the United States Government nor any agency thereof, nor any of their employces, makes any wasranty, express or implied, or assumes any legal liability or responsibility for the accuracy, completeness, or usefulness of any information, apparalus, proxtuct, or precess disclosed, or represents that its use would not infringe privately owned rights. Reference herein to any specific commercial product, process, or service by trade name, trademark, manufacturer, or otherwise does not necessarily constitute or imply its endorsement, recom. mendation, or favoring by the United States Government or any agency thereof. The views and opinions of authors expressed herein do not necessarily state or reflect those of the United States Government or any agency thereof.

\section{SELECTED MONTHEY OPERATING DATA FOR B AND T PLANTS, REDOX AND PUREX (1944-1972)}

\section{Compiled by}

\section{S.P. Gydesen}

April 1992

Distribution
1. Office of Scientific and Technical Information, Oak Ridge,
2. Tennessee
3. Richland Reading Room
4-6. S.M. Heeb
7. PNL-CMCC


The attached declassified pages (3-16) were extracted from HW-63089 "Chemical Processing Statistics - Book I, 1994-1972" Daye McDonald. The notebook was issued on December 15, 1959. The only information of potential interest and/or use to the Hanford Environmental Dose Reconstruction (HEDR) Project is the monthly data relative to operations at $\mathrm{T}$ Plant and Purex from startup to shutdown.

Declassified pages (17-27) were extracted from HW-63090 "Chemical Processing Statistics - Book II, 1945-1972" Dave McDonald, December 15, 1959. The only information in Book II of potential interest and/or use to HEDR is the monthly operating data which addresses B Plant and REDOX form startup to shutdown.

Since both of the notebooks from which these pages were extracted contain information on weapon part fabrication and shipment, they remain classified SECRET - RESTRICTED DATA. Individuals with the appropriate level of clearance and a need-to-know may contact the Westinghouse Hanford Company/Boeing Computer Service Richland - Information Resources Management if they wish to request access to these documents. 


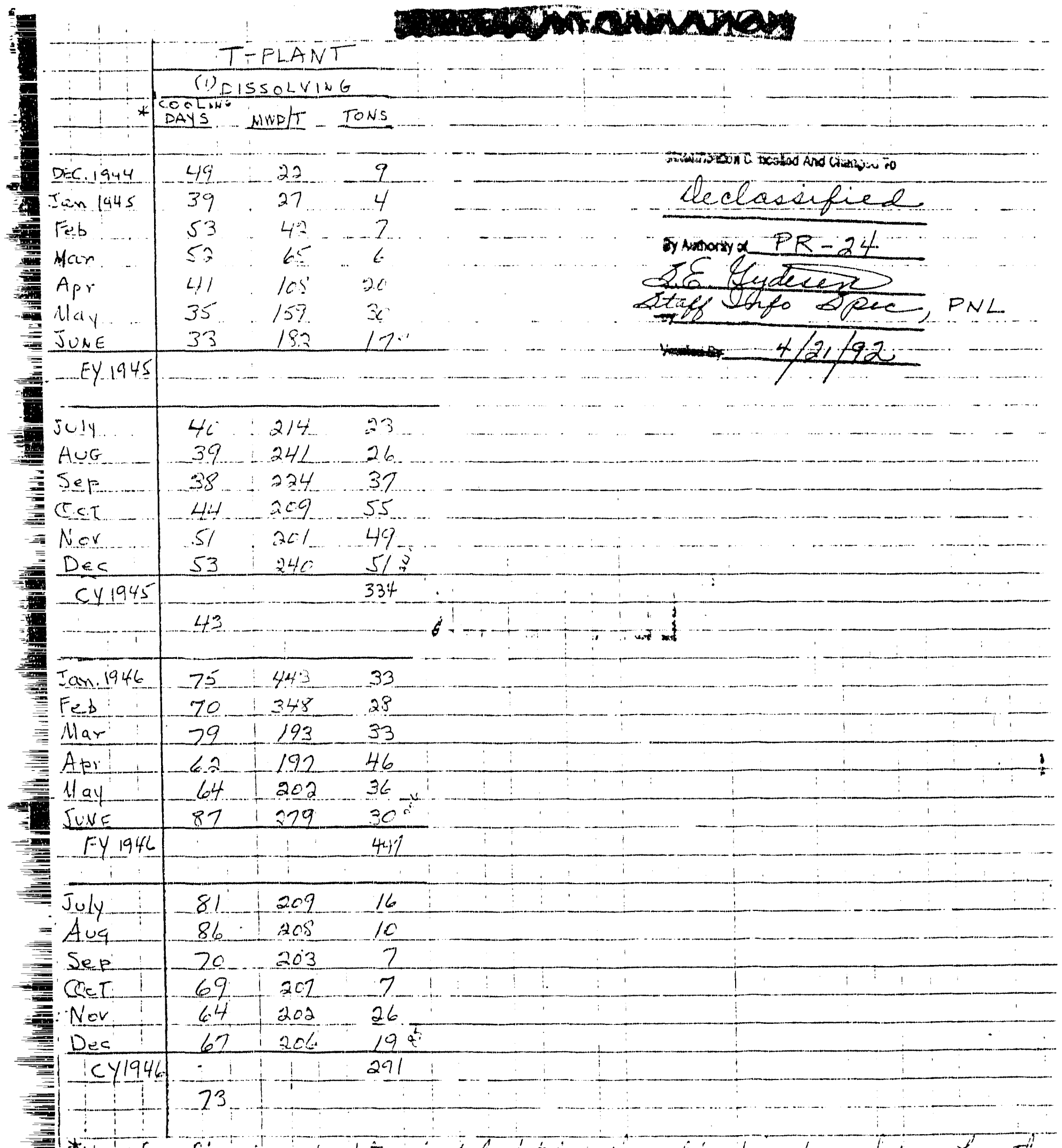

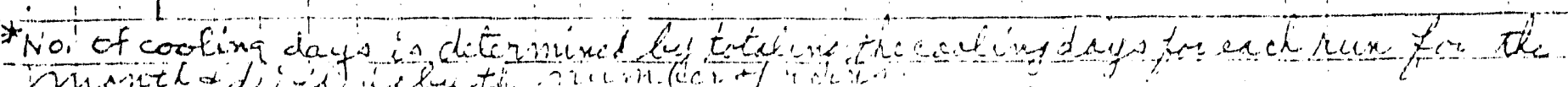

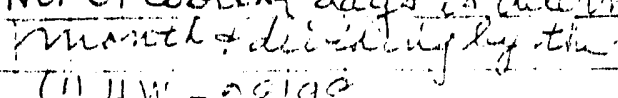
(i) $H$ W -399 


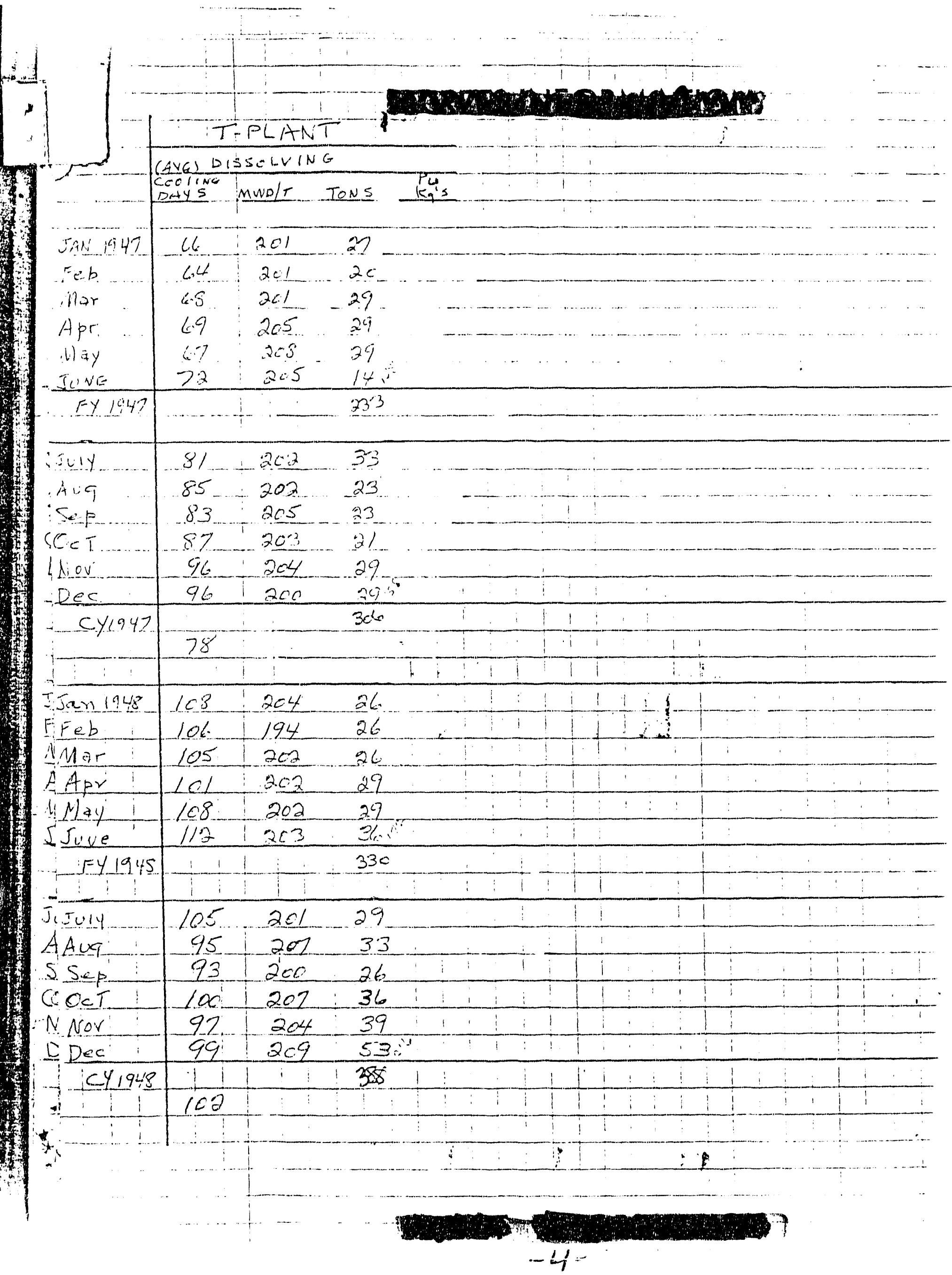


6

-1:i $\mid \frac{1-P L A N I}{1 \text { DISSOLVHO }}$ Ara. CeCline

Rays inWD/T ToWs

n $1949-961124$

$\because 43 \quad 263 \quad 46$

ar........ $93.2 \% 1 \quad 63$

pr $\quad 95 \quad 229 \quad 25$

lay $\quad \begin{array}{ll}94 & 248 \quad \therefore 6\end{array}$

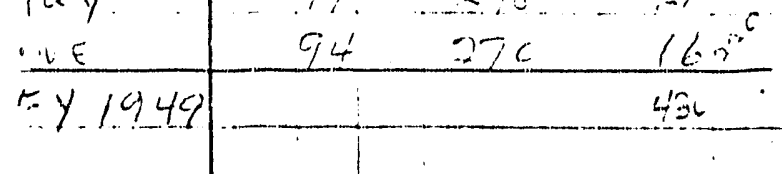

$214-46,288=0$

$.9 \ldots .65 \quad 275 \quad 26$

EF $\quad 94 \quad 323 \quad 26$

$T \quad-93 \quad-378 \quad 33$

cv $95 \quad 358 \quad 66$

\begin{tabular}{l|lll} 
ic: & 95 & 396 & 32 \\
\hline cy 1949 & 1.1 & 343
\end{tabular}

95

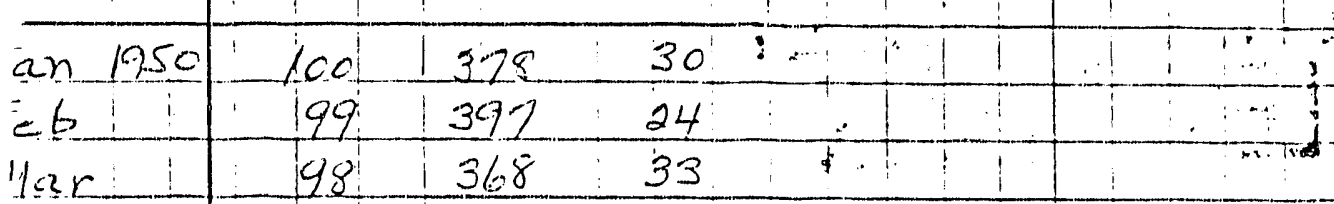

\begin{tabular}{l|l|ll} 
Her & 198 & 368 & 33 \\
\hline $1 p r$ & 99 & 352 & 36
\end{tabular}

May :96:372:30

\begin{tabular}{l|llll} 
Line & 98 & 473 & 20 \\
\hline Fy 195C & & 346
\end{tabular}

Uly : 9 i 472 38

c.9. 73 40s 49

\begin{tabular}{l|l|l|l|} 
ep 1 & 74 & 422 & 43 \\
\hline EI & 80 & 416 & 43
\end{tabular}

$6 \square+1 \quad 83 \quad 1532,36$

Jec I $75 \quad 7255 \quad 426$

cy $1950 \quad \frac{1}{89}: \frac{1}{124}$

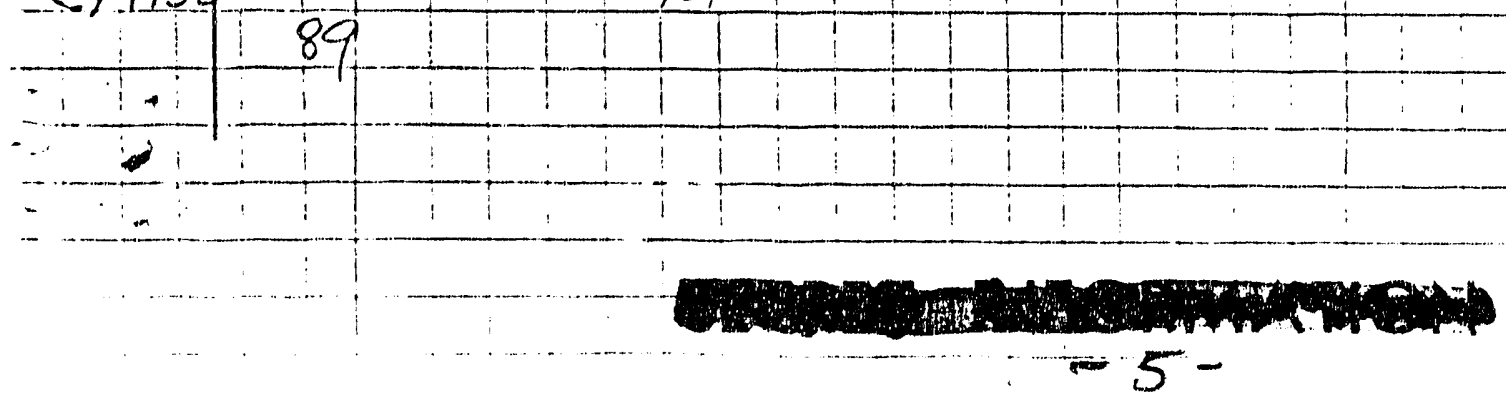




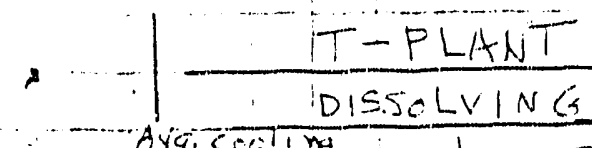

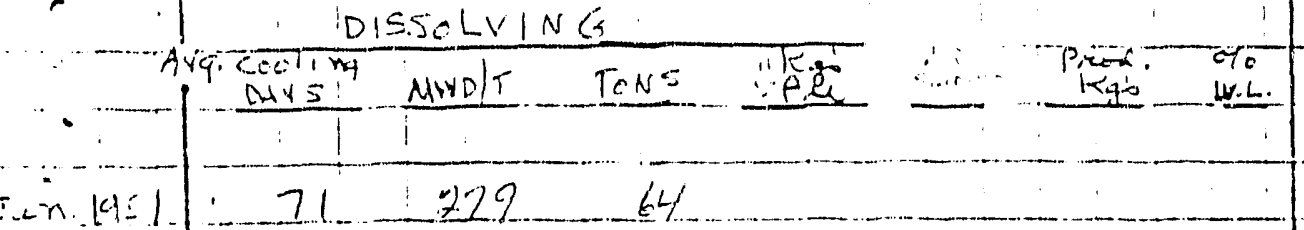

$\therefore t$

la

AfF.

lay

LA.

$6 y 1051$

$71 \quad 229 \quad 64$

$72 \quad 414 \quad 43$

$71 \quad 443 \quad 53$

$54 \quad 414 \quad 53$

19.01

$45 \ldots+411 \ldots+54.18 .73$

$47 \quad 415 \quad \frac{49}{66} 17,45$

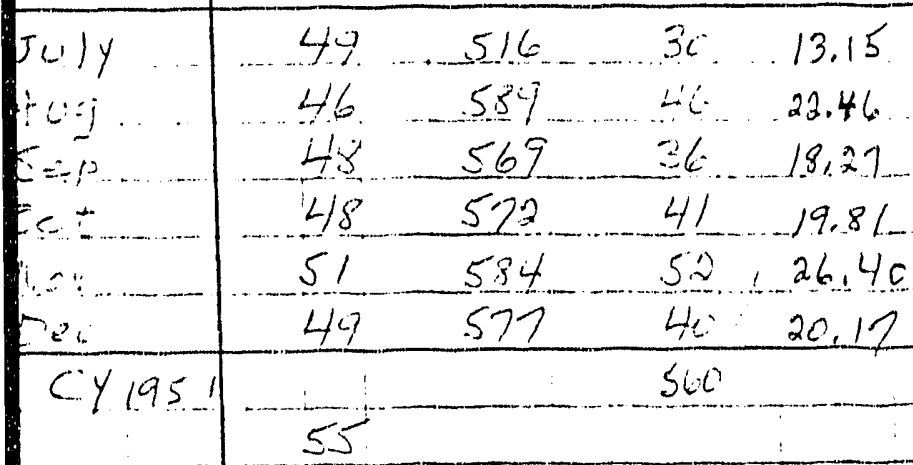

$\operatorname{sich.1952}$

$47 \quad 56 i \quad 53$

$50 \quad 587 \quad 26$

llar

Apr

lity

In ne

Fy $19=2$

$\begin{array}{lll}48 & 588 & 49\end{array}$

$51 \quad 552 \quad 33$

\begin{tabular}{l|ll}
52 & 602 & 16 \\
77 & 624 & 74
\end{tabular}

$4 \quad 4 x 7$

PSgD

$5 \cup 14$

$4 \cup g$

Esp

ret

Nór

Dé.

641959

$20 \frac{593}{1601}-\frac{30}{26}$

$83-6223$

$99 \quad 540 \quad 13$

$76 \quad 609: 13$

$6 c \quad 633$

$-\frac{65}{1}$

$20.6 ?$

17.31

$2 / 36$

22.10

14.49

$6.49 .0^{4}$

$29 !$

10.05

15.97

2.91

16.76

7.48

12.136

157.72 
T-PLANT

\section{monmm}

DISSOLVINE

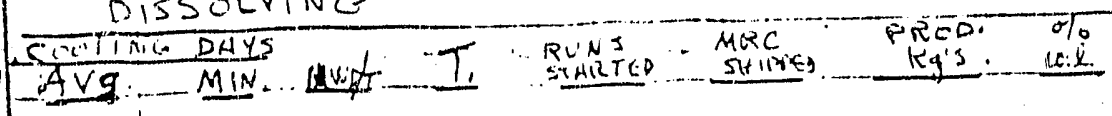

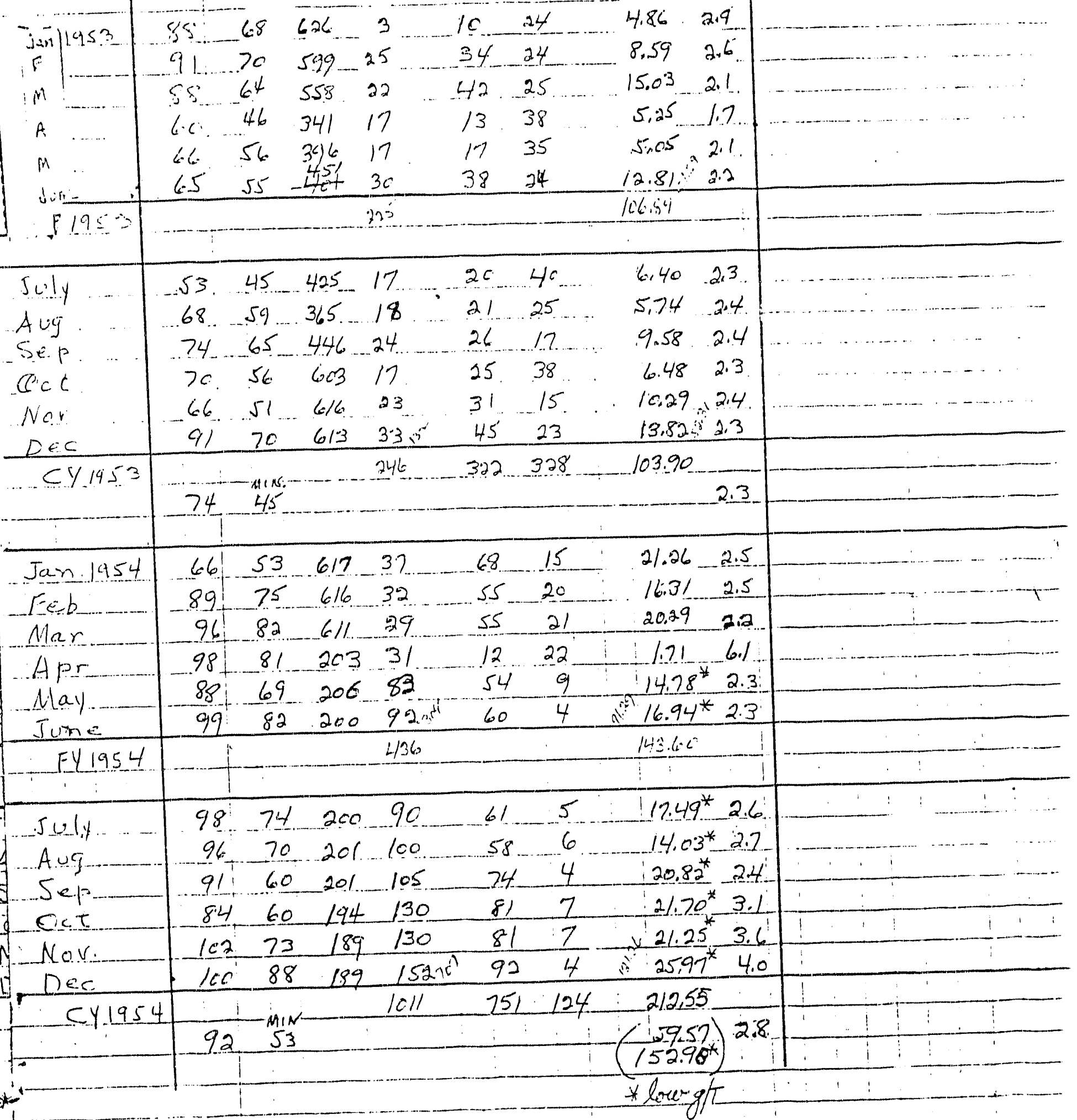




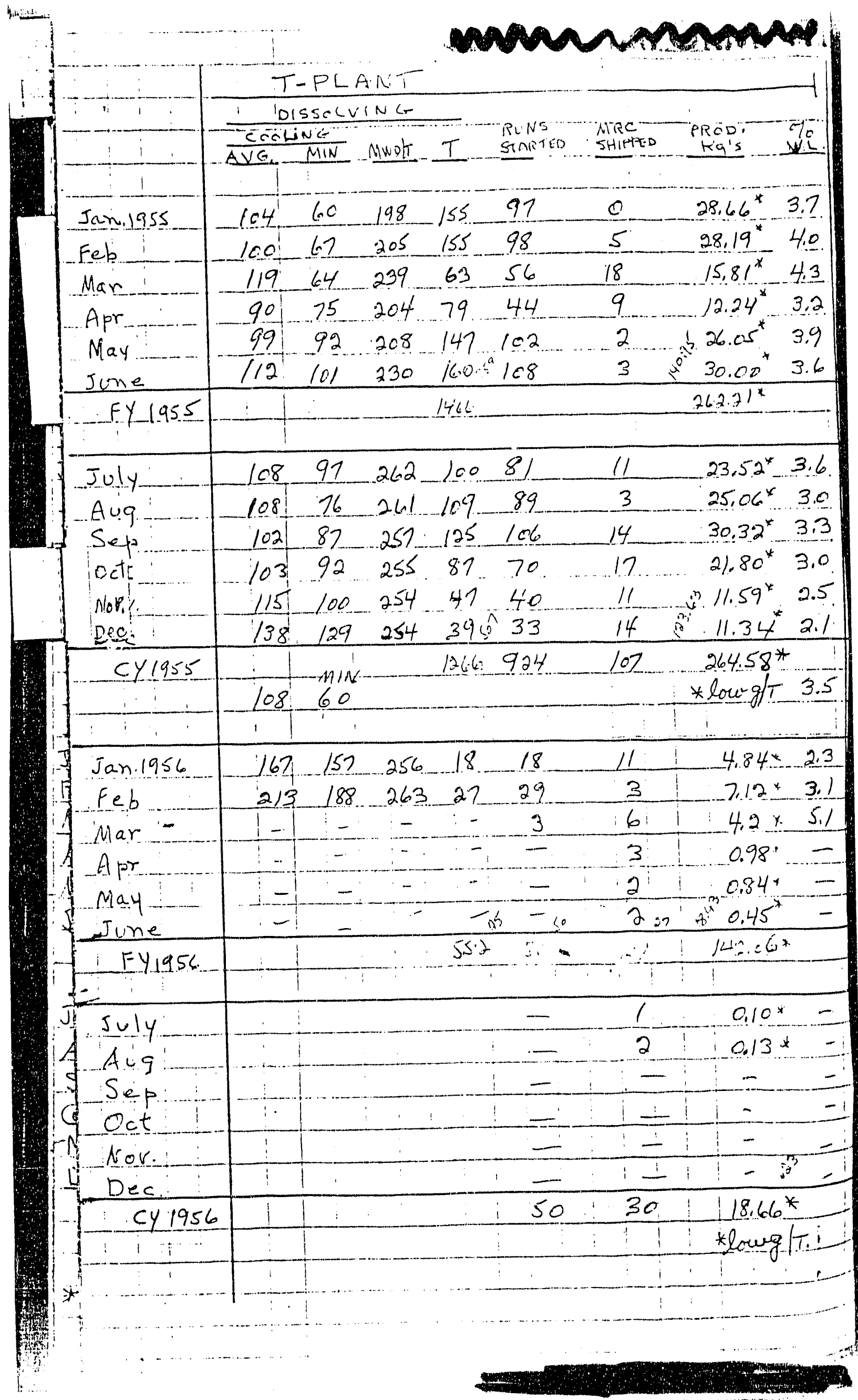

$\therefore$

TONS Kg's

$-67.03^{(1)} 12.53^{*}$ $246.22 \quad 53.60^{*}$ $136.92 \quad 21.27^{*}$ $196.85 \quad 58.52^{*}$ $132.95 \quad \therefore 27.24$ $341.14 \approx 73.55^{*}$

$1271-1924+4$ $1.2 .72+25.46$ $239.52 \quad 150.76$ $315.10 \quad 192.22$ $186.03-220.60$ $272.22 \quad 162.98$ $279.15 * 181.81$ $520.39 / 10578$ 839.33 266.45

(1) First month

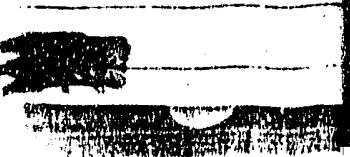




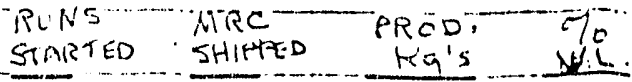

$5 \quad 97 \quad 0 \quad 28.66^{*} \quad 3.7$

$\begin{array}{lll}5 & 98 & 5\end{array} \quad 28.19^{*} 4.0$

$\begin{array}{llll}3 & 56 & 18 & 15,81^{*} \quad 43\end{array}$

$9 \begin{array}{lll}44 & 9 & 12.24^{*} \quad 3,2\end{array}$

$7 \ldots 102 \ldots \ldots 2 \ldots .026 .9$

$0.408 \quad 3 \quad 30.00^{*} 3.6$ $26.2 .7 \%$

0 8

$11 \quad 23.52^{*}-3.6$

3. 89

$3 \quad 25,06^{*} 3.0$

5.106

$14 \quad 30.32^{*} 3.3$

$17 \ldots \ldots .21 .80^{*} 3.0$

$1 \quad 40 \quad 11 \quad n^{n} 11.59^{*} 2.5$

$9533 \quad 14 \quad 11.34^{*} 2.1$

$6924 \quad 107 \quad 26458^{*}$ * lou g/t 3.5

$18 \quad 11 \quad 4.84 * 23$

$29 \quad 3 \quad 7.12 * 3.1$

$3 \quad 6 \quad 4.2 \times 5.1$

- $3 \quad 0.98^{\circ}-$

$-\quad 210.344-$

${ }_{0}=0227+0.45=$

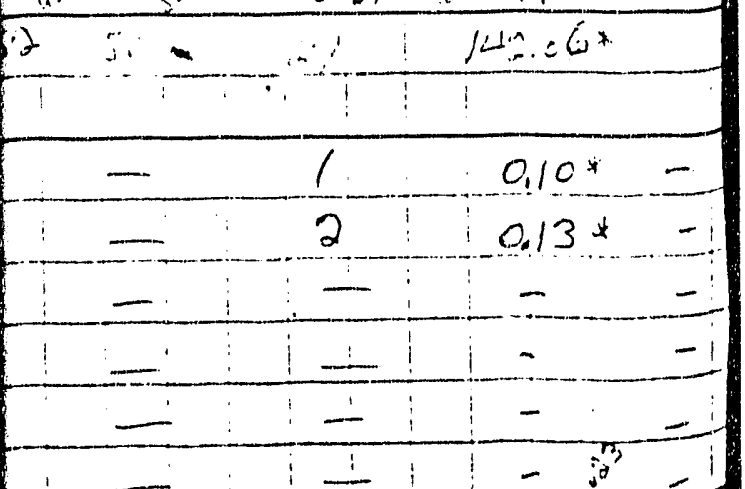

$50: 30: 18.66^{*}$ *lowg $(T, i$ bin

PUREX

TONS KG'S Down AVG $\frac{\operatorname{coL} I N G}{}$ WASTE LOS

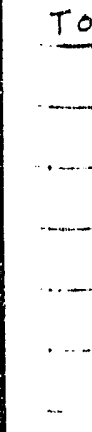

\section{$-61.03^{(1)} 12.53^{*(1)} 0151 / 38$} $246.72 \quad 53.60^{*} 31 \quad 181$ 145 $136.92 \quad 21.27^{*} \cdot 58 \quad 163 \quad$ III $196.85 \quad 58.52^{*}-40 \quad 10595$ $132.95 \approx 27.24^{*}: 55 \quad 122 \quad / 15$ 341.14 i: $73.55^{*}$ : $101 \quad 91$

$112.7 L \cdot 192.24460 \quad 145 \quad 131$ $239.52 \quad 150,76 \quad 24,135 \quad 116$ $315.10 \quad 19222 \quad 13.3 \quad 1 / 8 \quad 109$ $186.03+120.60 \quad 45,2 / 11 \quad 104$ $272.22 \quad 16298 \quad 300 \quad 108 \quad 98$ $229.15 \times 181.81 \quad 37.6 \quad 114 \quad 100$ $520,39 / 10578$

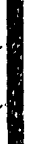

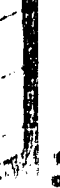

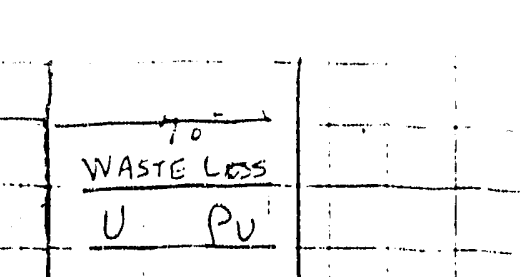




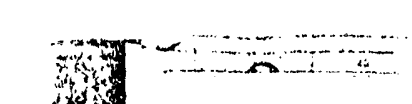




\section{PIREX}

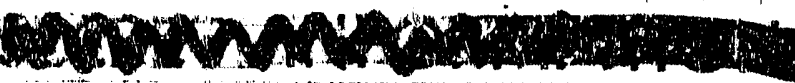

TONS KG' DONO $326.50 \quad 1.9302 \quad 386 \quad 104 \quad 81 \quad 12 \quad .29$ $291.36 \quad 166.26 \quad 540,105 \quad 91 \quad 106 \quad 19$

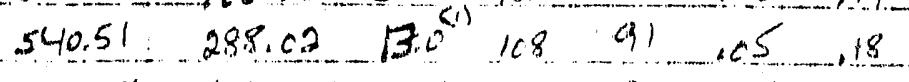
APR … $\because 270.64 \quad .116 .03 \quad 17.5 \quad 107 \quad 93 \quad .14 \quad .53$

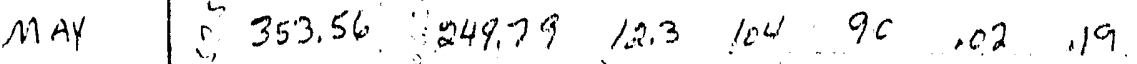

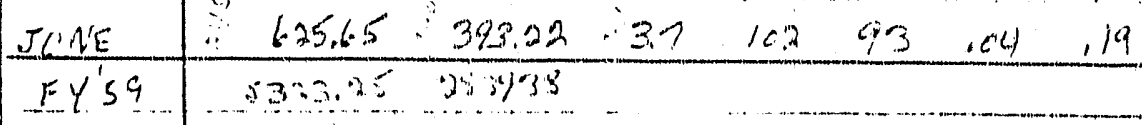
IULy 21486.1440237 .1110 87 18 $.706 \quad 608.99408 .68$ o $1,91.05,13$ SEP $\quad 44472 \quad 312.97 \quad 160 \quad 9589.10 \quad 18$

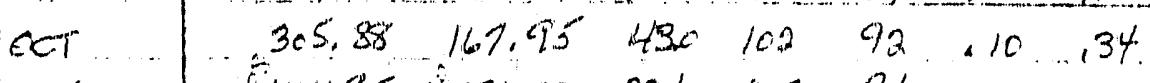
sov ... 441.95 . 274.32 33.6 107 91 .09 .28

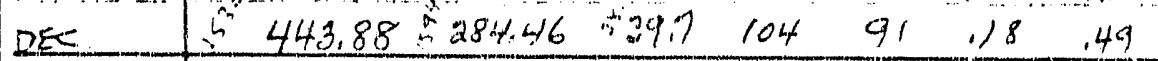
cy'59 498764360074 av6 1026

196.0

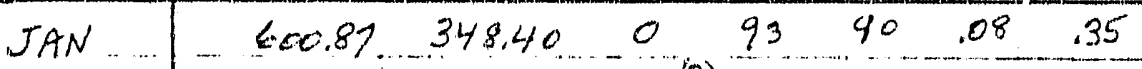
EEB $\quad 37569 \quad 251.46 \quad 299^{(0)} / 00 \quad 92 \quad 10 \quad 39$

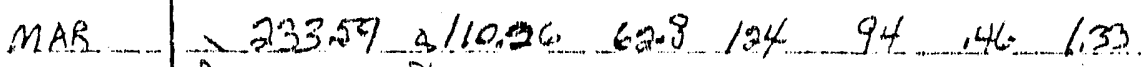
APR : $\quad \begin{gathered}\text { N } 72717 \\ \text { N }\end{gathered}$

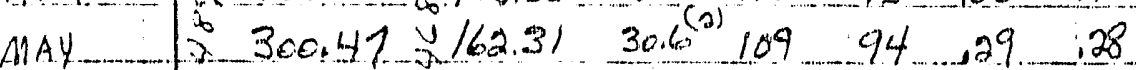
$\begin{array}{llllllllll}\text { IUNE } & 0 & 25192 & 143.53 & 67.4 & 122 & 103 & .28 & 1.83\end{array}$ Fy' $50 \quad 5019.1993062 .68$

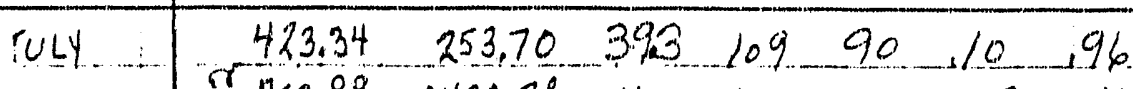

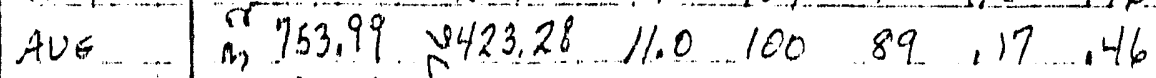

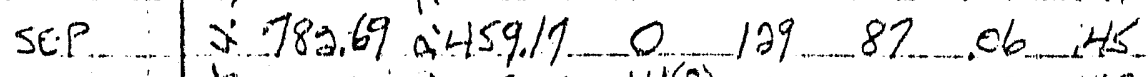
CET: $\quad 403301205.27 \quad 142) 197 \quad 101.08 \quad 43$ Nor $\quad 688.08938 .24 \quad 0 \quad 11790 \quad 16 \quad 148$ \begin{tabular}{l|lllllll} 
DEE & 709.93 & 508.60 & 15.6 & 103 & 91 & .09 & .36 \\
\hline CY 60 & 6244.03 & 3647.04 & & & & & 50
\end{tabular}

\section{(1) $20 y$ palm} apteting ture

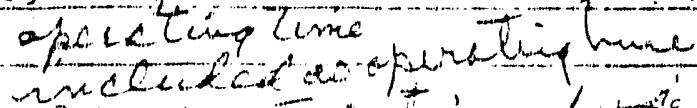

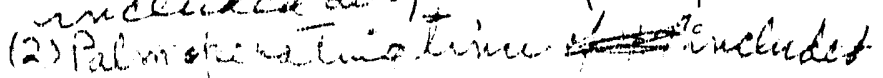




\section{PUREX}

Secting WHSTELos

TON'S Ka's Jo AVG MIN U Pu

1461

SAN

FEB

MLAR.

Apr

MAY

Junif-

$\begin{array}{llllllll}33 / 71 & 180.81 & 28.6 & 14 & 93 & .18 & 52\end{array}$ 047492 - $292.29 \quad 33.4$ (11 92 . 09.30 $2560.72 .358 .90 \quad 7.5118 \quad 96$ il6 136

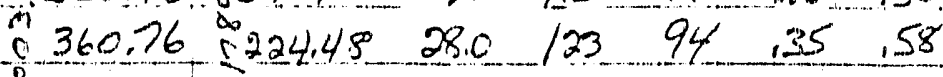
$\therefore 694.95 \quad 429.31 \quad 12.4 \quad 124 \quad 96,09 \ldots .25$ Ey $1961 \quad 6757.80 \quad 3968.56$

\begin{tabular}{|c|c|}
\hline 504.4. & 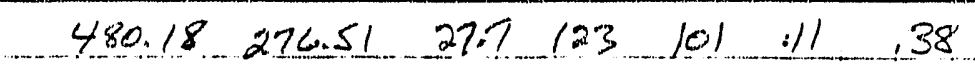 \\
\hline AUCE & $\cdot L 634.63 \quad 349.88 \quad 10,0 \quad 115 \quad 104,20^{1}, 36$ \\
\hline SEP & $8496.543269 .29 \quad 33.1 / 21 \quad 110.16 .34$ \\
\hline$C T$ & $5371.42 \quad 188.23 \quad 558 \quad 114 \quad 100 \quad 18 \quad .37$ \\
\hline 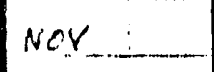 & $282.11=166.70 \quad 52.6 \quad 116 \quad 106.18 \quad .24$ \\
\hline 三 & $678.87 \quad 440.79 \quad 5.6 \quad 166 \quad 112 \quad .10 \quad 13$ \\
\hline 6.1961 & 5947233501.70 \\
\hline & $-5 q u 1 T$ \\
\hline 1962 & 1 \\
\hline JAN & $\begin{array}{lllll}313.29 & 197.45 \quad 29.5 \quad 142 \quad 132 & 127 \quad .44\end{array}$ \\
\hline$F E B$ & ${ }_{-\infty} 14 t ? 99-66.89 \quad 2460-145 \quad 130 \quad .61 \quad 157$ \\
\hline MAR & $=305,64: 213.83 \quad 36.4 \quad 162 \quad 121 \quad 16 \quad 120$ \\
\hline$A P R$ & $\therefore 302.39 \mathrm{~m} 192.79 \quad 340 \quad 174 \quad 150$ 35 64 \\
\hline MAY & $\begin{array}{lllllll}620.56 & 338.97 & 0.6 & 162 \quad 125 & 0.38 & 6.37\end{array}$ \\
\hline JUNE & $\begin{array}{llll}15.6 & 156 & 126 & 131 \\
\end{array}$ \\
\hline EY 1962 & $5185.67-3459.16$ \\
\hline & $11 \div 11 ; i 1$ \\
\hline Juty 1 & $776.79,412.89 \quad 0.6-142 \quad 120 \quad 131 \quad 137$ \\
\hline Aus & $271.05157 .78 \quad 45,136 \quad 120 \quad 338 \quad 70$ \\
\hline SEET & $\therefore 498.97 \quad 0287.69 \quad 21.6 \quad 199 \quad 131 \quad 36$ \\
\hline CCT & $\therefore 772.430445 .99 \quad 161 \quad 155 \quad 122 \quad .24$ \\
\hline NoV: & $695019350.50 \quad 177^{1} 153$ ily 127 \\
\hline DEC & $\begin{array}{lllllll}78 & 4.54 & 448.15 & 6.5 & 127 & 107 & .31\end{array}$ \\
\hline$C Y 1962$ & $6040.813450 .26+1$ \\
\hline & $K=1$ \\
\hline & 1 \\
\hline
\end{tabular}




\section{PUREY}

\section{TONS Kis 1.0}

1963

IAN

FE BS.

- MAR

APR.

NMAY

EUNE

Fy 1963

三

JUI.Y

$A \cup G$

SEPT.

EOT.

$\equiv$ Nov,

DEC

cy 1963

$=$

1964

TAN

$\equiv$ FEB

$=N A R$

$=A P R$

MAL:

IUNE
9.36

0

के $867.24,0477.22$

in 1.9 .12 के 39.09

609.07 .370 .97

273.90470 .6

6537.113690 .79
405.620 .230 .05

\section{Fy. 1964 \\ 5218.86323490}

COOLING. WASTELESS

Avé Min U.... Pu
100

38,0

5.0

.24 .38

$94.0 \quad 136 \quad 130 \quad 5.3 \quad 2.9$

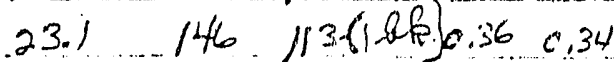

$1 / 57 \quad 149 / 19(16 b) .22 .33$

\begin{tabular}{|c|c|c|c|}
\hline $12.7 \% \ldots 6.97$ & 100 & - & $3,274.19$ \\
\hline or $=0280103.96$ & 54 & 155 & 124 \\
\hline 631.290356 .16 & 0 & 154 & $21^{*}$ \\
\hline
\end{tabular}

$\therefore 453,563353,24 \quad 11.3 \ldots 160120 \quad .25 \quad 36$

JULY $\quad 289.29127 .75: 49: 148 / 21 \quad .26 \quad 50$

AUG $\quad 856.19483 .35 \quad 01 \quad 139-121.22 .28$

SERT $20762 \pi 38 / 19 \cdot 08 \quad 228 / 66 \quad 25 \quad 45$

OCT 242852456.28 o6 $137 / 21 \quad 27.32$

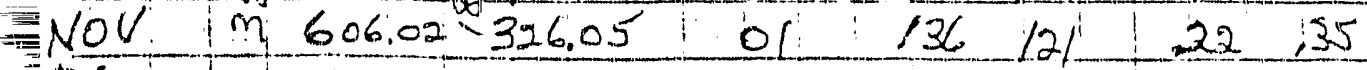

DEE: I $117.66 \quad 65.69,79 \quad 139123 \quad .40 \quad 1.28$

$=$ EY $1964: 5759,203459.35$

$1890.30 \%$ TinD

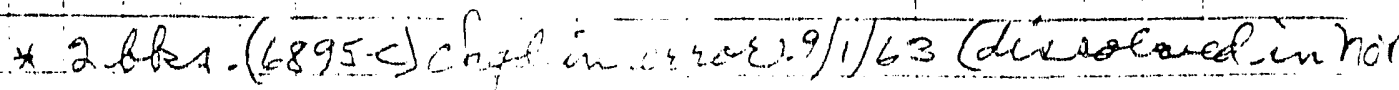




\section{PUREX}

COOLING: \%OWATELLOSS

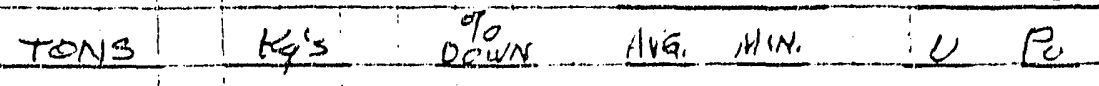

1965

TAN

VES

WAR

APR

NAL

JUAE

7419656737.963790 .33
$(.24)$

$0390.62 \sqrt{2} 28.34 \quad 50$

$00807.07 .406 .90 \ldots 15$

$-908.510496 .09 \quad 02$

$883.20 \div 466.54$

$599.44 \quad 302.15$

0

24
$=-$

$169+131$

$169 \cdot 120$

$186-122$

157.125

$128 \quad 125$
0.380 .43

$.29 \ldots .30$

$.24 \quad .32$

$.25 \quad \ldots .29$

$.36 \quad .51$

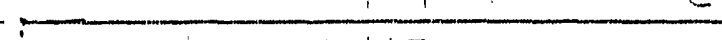

$=212 y$

$262 ! 18 \cdot 141.28$

6

$458,86,28899$

19

165126

$167-119$

45

133

.45

Al's.

$050638 \sqrt{26788}$

23

$175 \ldots 131$

1.23

139

CCT,

if $388,66,205,5.5 * *$

14

$-14) \quad 119$

22

152

Nor.

210,64

12565

56

163192

$137 \quad 122$

Cy $1965 \quad 5770.43 \quad 3176.78$

$$
3=39.36 a c
$$

1966

JAN

YEE

$4 / 299236.75 \quad 31$ $230,75-16$

$6 \quad 190<25$

30 46

27

$12 \%$

, 56

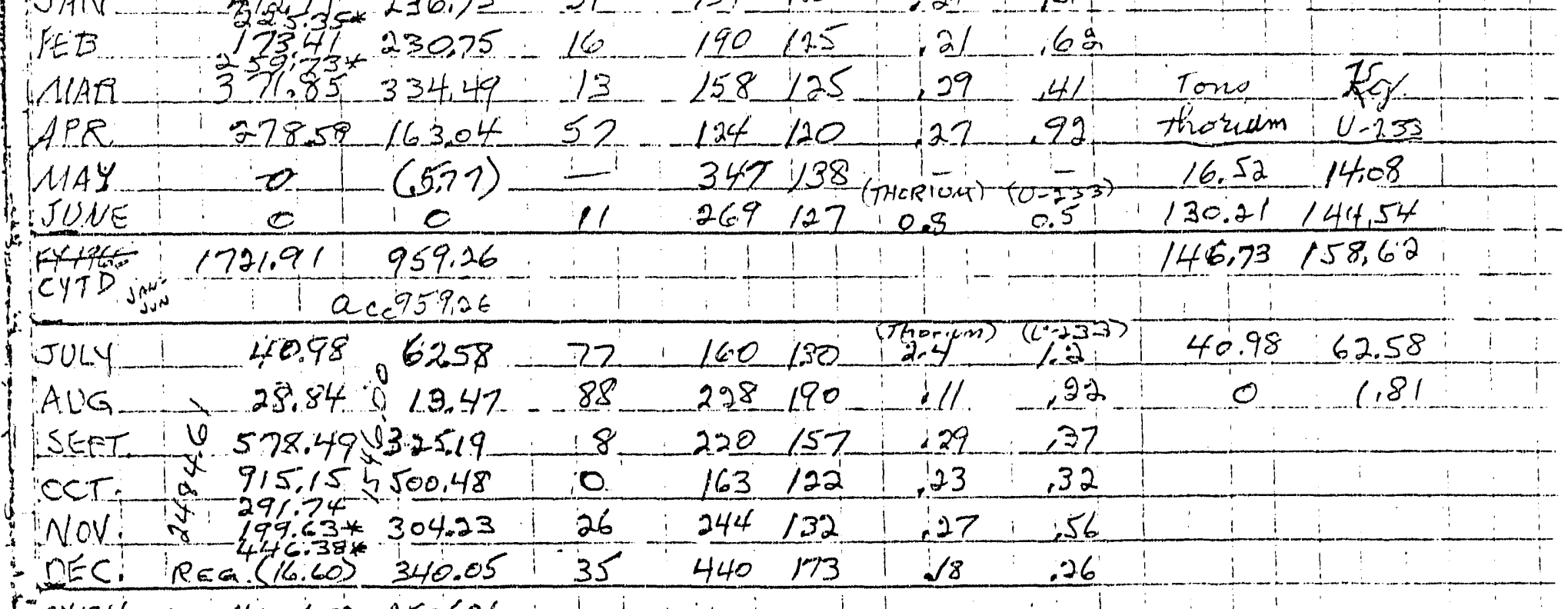

Cy1966 an 420652 2505.26

$$
\begin{aligned}
& * 62.588 \text { transers } \rightarrow A \\
& * \text { Enrich }
\end{aligned}
$$


알
$=$
$=$

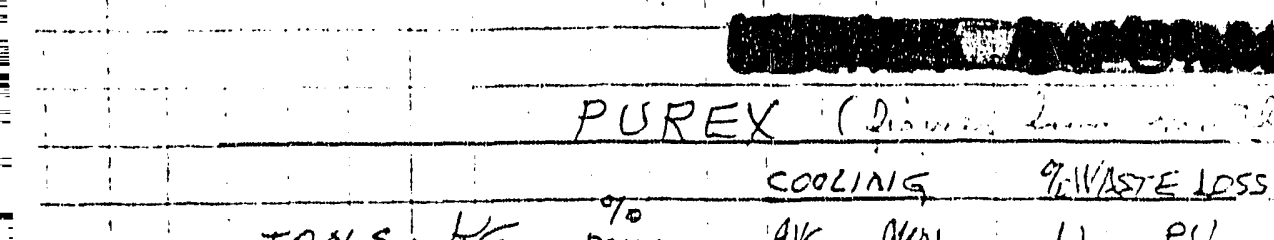

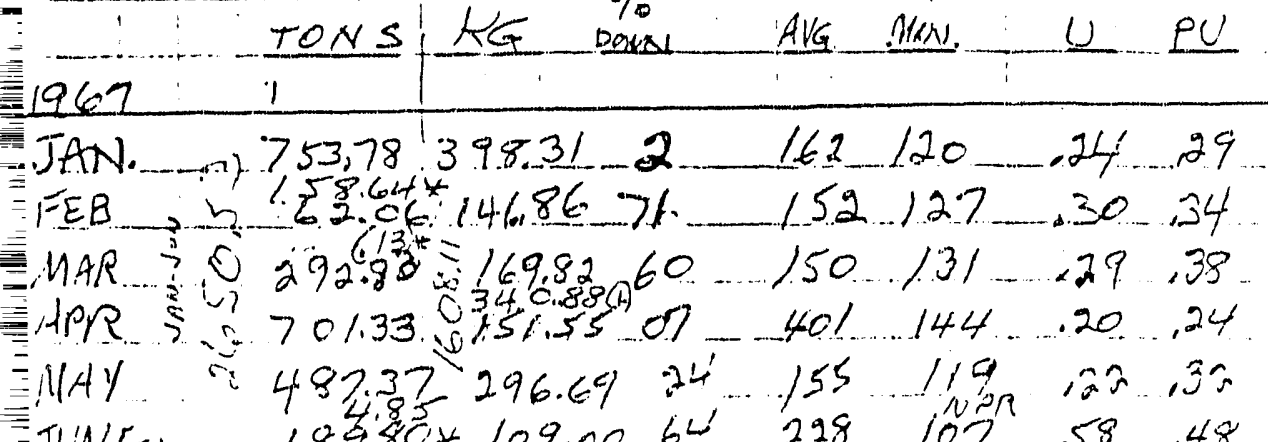

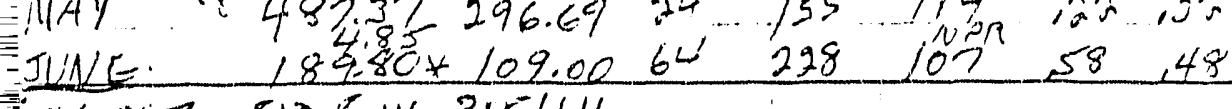
$\begin{array}{lll}1.962 & 5135.14 \quad 315411\end{array}$

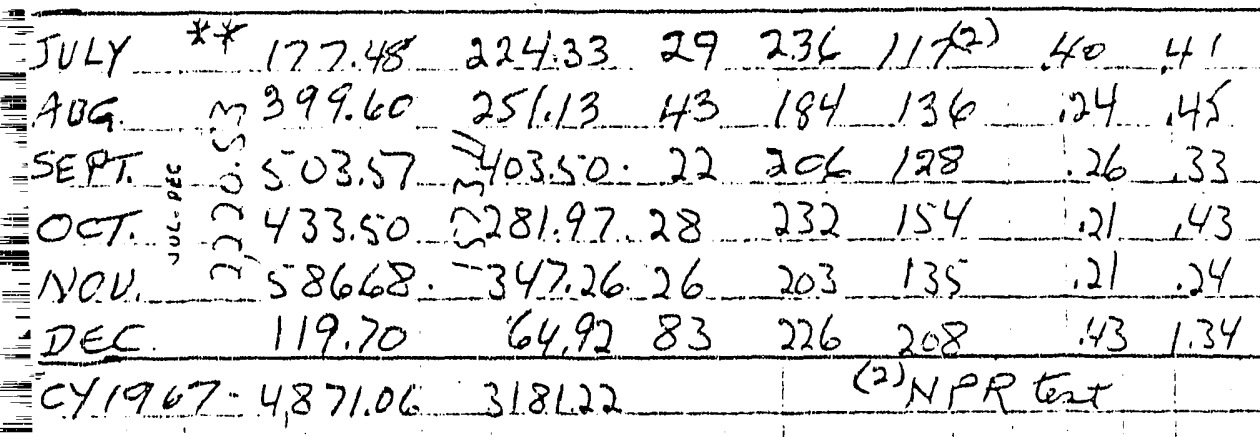

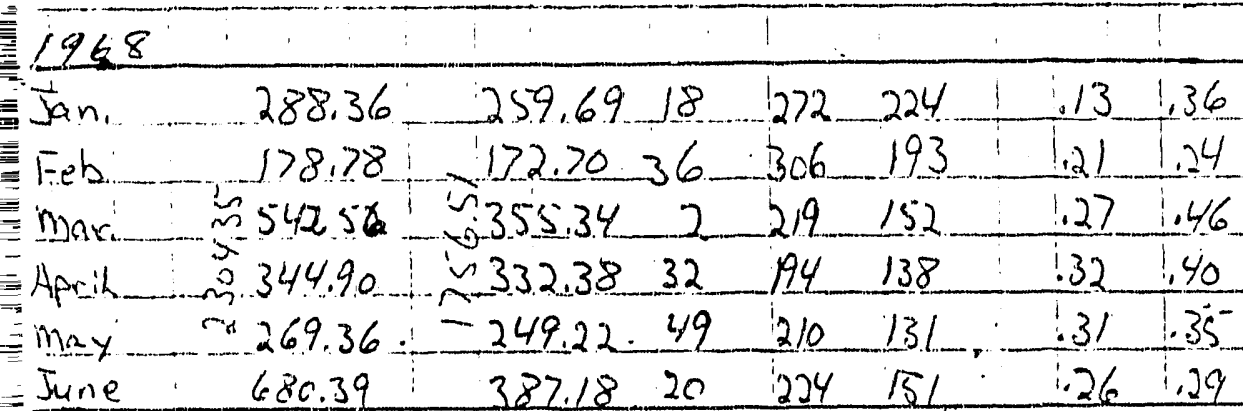

FY $9^{2} 66^{\prime}-4529.88-3,329.62$

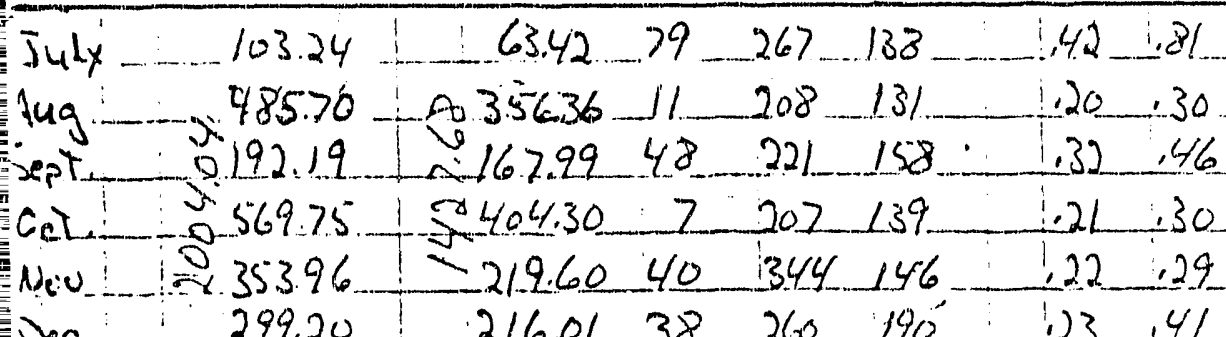

\begin{tabular}{ll|llllll} 
Dec & 299.20 & 216.01 & 38 & 263 & $190^{\circ}$ & 123 & 141
\end{tabular} cy $1968-430839+3,18419$

* Enriched

(1.) now-deferse

* zircalon-N Reator 135

$-14-$ 


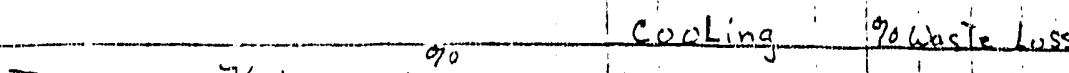

1969

Tons Kg's ocow Avg min.

Jan:

$0 \quad 6.05+289$

$244 \quad 230$

Feb.

$128.38-263.57 \ldots-23$

$297 \quad 195$

mav.

346.09

$\stackrel{\sim}{\sim} 316.94$

$247 \quad 184$

$34 \quad .46$

Apr.

$=270.0$

May

459.90

$\doteq 173.16$

37

300

284.57

23

37

234

183

$.19 \quad .24$

June 109.52

EY 1969

$231221 \quad 252220$

JuLy.

Aug.

Sept.

OCT.

Nou.

Dec

cy 1969

Jan.

Feb.

mar

Apr.

may

June

FY 1970

Juliy

Aug.

Sept.

OcT

.368 .62

384.38

95.23

35.296

25.14164

$\frac{51.98}{2476.32-205022}$

.17 .67

24.81

$318.80 \ldots 24$

21

29

37

303.45

318.42
190.47

204.35

.23
222

181

$.22-.39$

$.33 \quad .48$

$215 \ldots .189$

$1.49-1.88$

$261 \quad 180$

$30 \ldots 33$

$\begin{array}{llll}226 & \ldots 175 & .27 & .24\end{array}$

$244 \quad 187$.

$291 \ldots 191$

$361 \quad 242$
38 so

$.25 \quad .46$

$20 \quad 22$ nou

Déc

cy 1970
11.99 .94

.56

215

246.37

62.28

84.27

$\therefore 955 \div$

$9 \begin{aligned} & 9.55 \quad 5 \quad 17.23 \quad 88 \\ & -311.61\end{aligned}$

88

$-0$

277

326

$61.93 \ldots 131.23$

62

47.83

82

$\div$

172

0

185

219

201

-

.

$-$

$-r$

$\begin{array}{r}-\quad 100 \\ -\quad 13 \\ \hline\end{array}$

\section{G 121.99. Jhominnv/33.19(4.233) 10}

28.01 Jhominn 58

155.06 .2 hominamis $110.20(4 \cdot 231) 18$ $89.525 h_{\min } 2257(4233) 56$ $1395^{\circ} \quad 438$ $983 \quad 355$ $585 \quad 358$ $534 \quad 303-2.5550 m-544(u=233) \div-$ $525 \quad 110$
.91.) 30 ming $3.22(4.233)$ 2.76 thim $10.86(4: 233)$ $4.6306-3.14\left(4^{\prime} \cdot 233\right)$ 


\section{$P U R E X$}

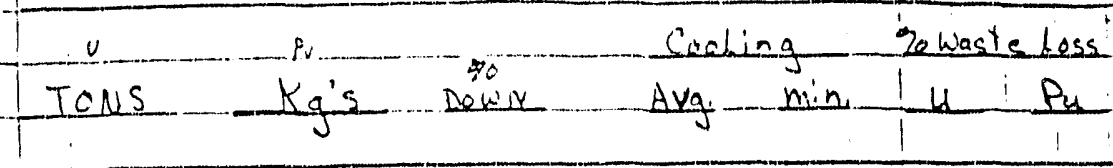

197

Jan

Feb. $! 1=$

$23.42(4.233)$ Eowh

mar.

Fin -

Apr

is 400.91

May

$\therefore 728.49$

अi3

49743

Dowis

June

$\frac{497.43}{345.65}$

FY 1.271

JuLy

Ang.

$16: 6.33$

$3=105$

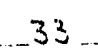

21

19

$=$

$-$

$-$

454355

$363-283 \quad \ldots .23 \quad 64$

$255 \quad 203 \quad 22 \quad .51$

Sept.

Oct.

$210.01 \ldots 1 / 343 \ldots \ldots$

$637 \quad 582 \ldots 42 \ldots 69$

Nou

$64751 \quad 37963$

$595 \quad 264$

$.25 \quad . .27$

m $627.94 \ldots 345.22$

$537 \ldots .233 \ldots .26 \quad \ldots .28$

Dec

in 77.49

总 44.02 .

$1024 \quad .272$

$.98 \quad 1.09$

C. Y $19 ? 1$

$122.76-157.94$

77

$31 \pm \quad 279$

$.53 \quad .61$

$237.66 \quad 362.3$

23

$767 \quad 311$

$.35 \quad .64$

$350020 \%=098.773213$

1972

JAN

FEb

Mar

$A P R$

May

90.32

$5 ? 66$

5

70.382

$419 \quad .71$

51.22

i. 575.86

$m 169: 1$
$\alpha$

$7 \%$

16

90

Q 25.49$)$ in (.01)

91.49

$(.67)$

16.09

65

11

FY $1972 \quad 2614.09 \quad 1906.20$

JuL

Aug

Sep.

OCT.

Nov.

Dec

Cy $1972 \quad 10.98 .42 \quad 105 \%$

$158.76 \quad 232.16-14$

$8206.90 \approx 289.26 \quad 0$

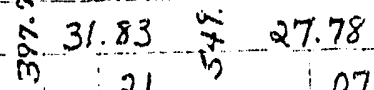

$9 \prod_{0 \rightarrow \infty}$

$\operatorname{Dom}_{\substack{a \rightarrow 3 \\ a-12}}$ \begin{tabular}{ll|l}
$413 \quad 370 \quad 62 \quad 82$ \\
\hline
\end{tabular}

$418 \quad 397$

$23 \quad 40$

$=\quad-\quad-\quad 1463$

$-\quad-1-100$

1.00

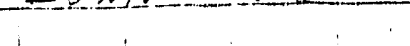




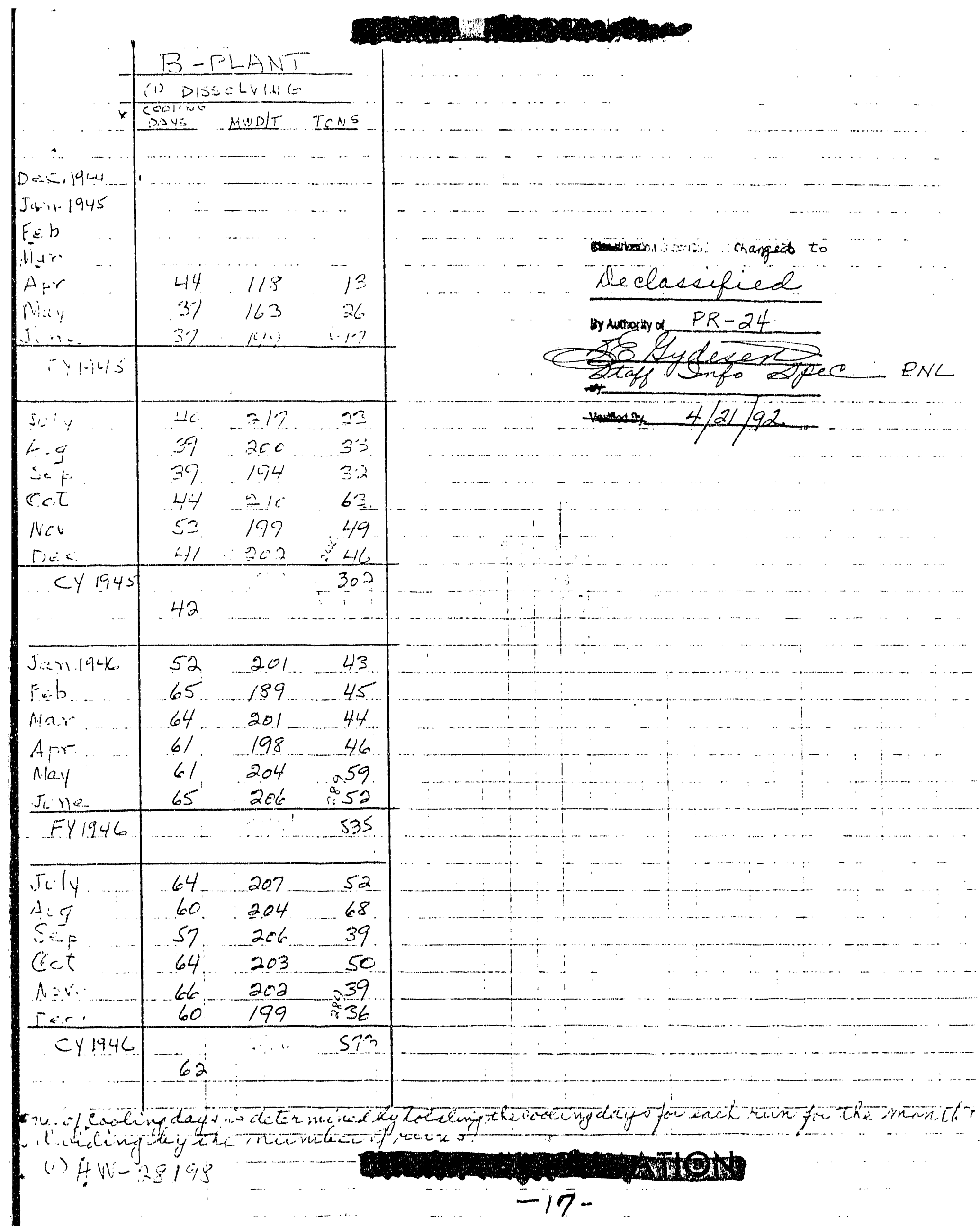


Cogling MWUIT TONS

$.5(27) .1947$

Feb

Mar

Apr

May

Jine

FY 1947

$59 \ldots 1.99$

33

$72 \ldots 201 \quad 26$

$69 \ldots 204 \ldots 33$

$68 \quad 206 \ldots 33$

6). $207.2^{29}$

75

20

\begin{tabular}{l|ccc}
\hline July & 75 & 205 & 29 \\
Acg & 79 & 205 & 29 \\
Sep & 34 & 201 & 33 \\
Cut & 94 & 203 & 20 \\
Nor . . & 94 & 202 & 23 \\
Dec & 94 & 201 & 427 \\
\hline Cy 1947 & & & 345 \\
\hline
\end{tabular}

Jan 1948 $99 \quad 200 \quad 32$

Feb

Mar.

Apr

May

June

Fy 1948

$100 \ldots 197 \ldots 36$

$106 \quad 201-26$

$109 \quad 241 \quad 27$

$120 \quad 233 \ldots 18$

- $: \ldots$

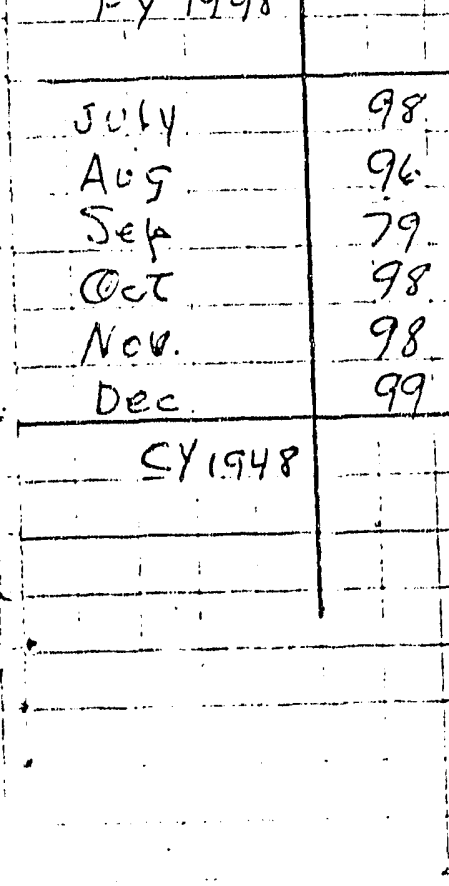

202

ก 39

301 
B-PLANT

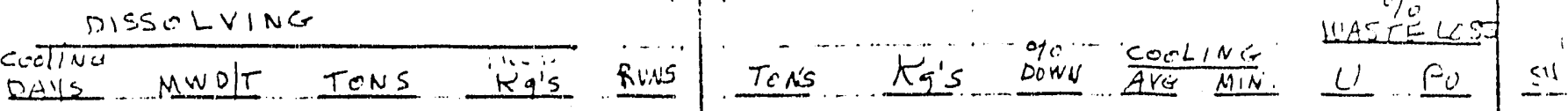

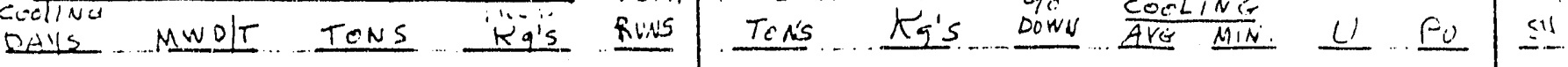

$89 \quad 408+53$

$77 \ldots 403 . \quad \ldots 49$

$62 \quad 413 \ldots 53$

$\begin{array}{llll}53 & 416 & 47 & 16.65\end{array}$

$50 \quad 420 \quad 53 \quad 20.36$

Sc $41.5 \quad \therefore 22 \quad 8.05$

J.p.

Fy 1951

JLIY

Aug

SEP

Sict.

Nov.

Des

Cy 1951

$56-508$

$\operatorname{Jan} \cdot 1952$

Feb

Mar

Apr

May

June

Fy 1952

$\begin{array}{llll}47 & 555 & 16 & 750\end{array}$

$48, \ldots 85 \quad .43 \quad 20.13$

$49 \quad 576 \quad 36 \quad 1794$

$\begin{array}{llll}46 & 567 & 46 & 22.33\end{array}$

REDAX

46. $592 \quad 53 \quad 26.53$

$\begin{array}{llll}51 & 526 & \because 37 & 18.62\end{array}$

PREDL $\angle E D$

Kqin RuNS

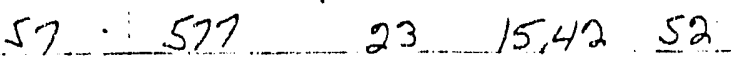

$64: 603 \quad 7 \quad 3.06 \quad 9$

$3: 64^{(1)} ?$

$22.66 \quad 5.43$

$-3.3$

$43.11 \quad 18.32$

4.5636

$74.99+31.70$

$62 \quad 603 \quad 2 \quad 43.81 \quad 5 \quad 578.08 \div 31.69$

$64 \div 579 \therefore 4 \div 3.87 \quad 10: 44.82 \div 27.08$

2.321834

$34+9$

(.............

-1

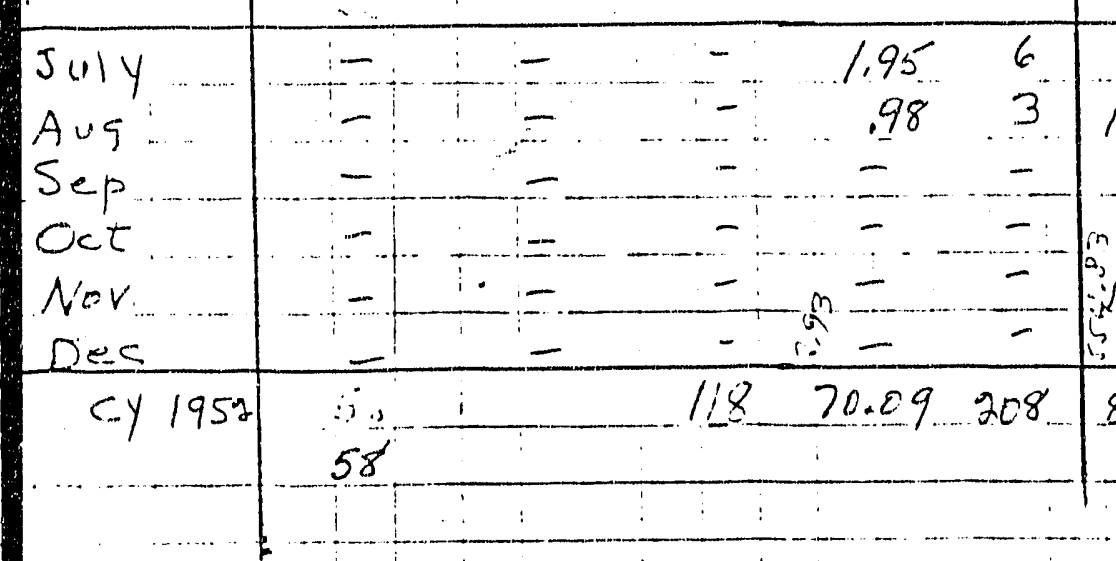

(1) First morith of operation 


\section{FEDOX}

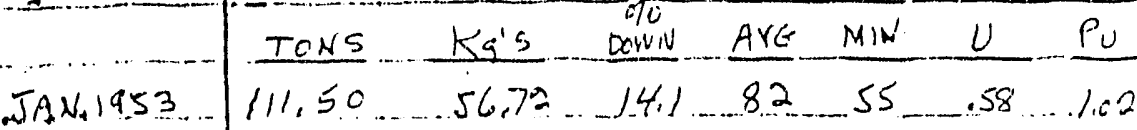

feb

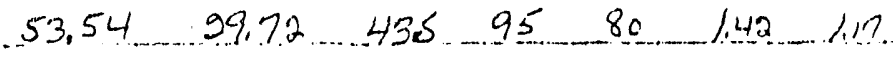

Mar

$\begin{array}{llllll}106.95 & 66.54 & 15.0 & 91 & 83 & 1.21\end{array}$

Apr

$132.14 \ldots .69 .65 \quad 12.2 \quad 83 \quad 75 \quad .78 \ldots 1.69$.

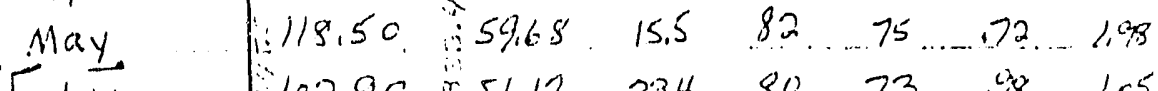

Ju'

$\begin{array}{llllll}102.90 & \times 51.17 \quad 33.4 \quad 80 & 73 & .90 & 1.05\end{array}$

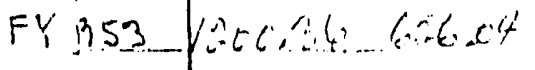

July $\begin{array}{llllllll}157,56 & 83.04 & 159 & 73 & 69 & 1.23 & 1.26\end{array}$

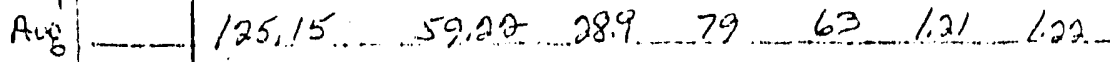

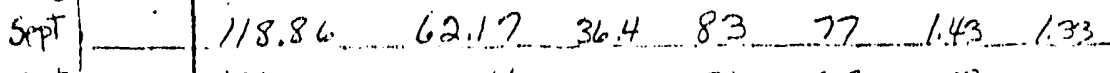

out

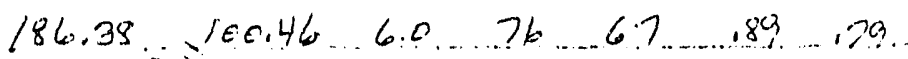

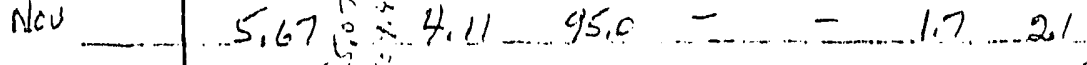

Dec _ $\quad(91.45 \%$

cy953. 1630.6 242.89

$26.8 \quad 81 \quad 5 \mathrm{SIN}-1.04 \quad 1.29$

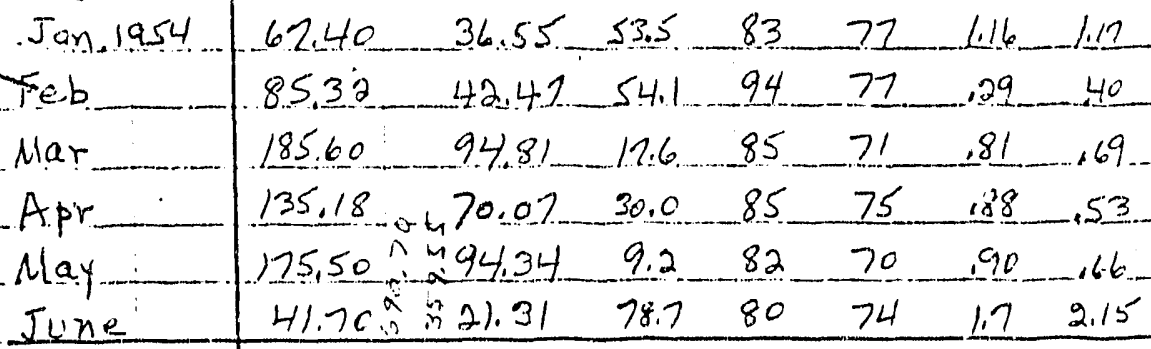

Fy $195414251276 \%$

\begin{tabular}{|c|c|}
\hline July & $40.10 \quad 571 \quad 106 \quad 76,47,73$ \\
\hline Aug. & 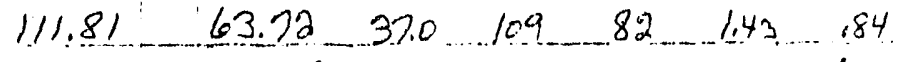 \\
\hline sep. & $154.09 \quad 86.25 \quad 18.0 \quad 10290 \quad 121.60$ \\
\hline $\mathbb{C}<\tau$ & $260 \quad 134 \quad 101 \quad 1.45 \quad .58$ \\
\hline Nov & 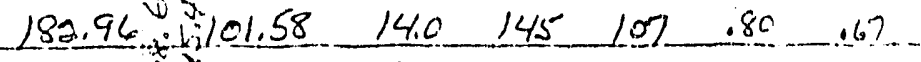 \\
\hline Dec & $215.57 .4 \div 115.18$ \\
\hline Cy 1954 & $15 \times 2.30 \quad 8.55 .04$ \\
\hline & $33: 2-106 \quad 7.0 \quad .95 \quad 70$ \\
\hline
\end{tabular}


REDOX

\begin{tabular}{|c|c|c|}
\hline & REDOX & \\
\hline & ConLING WASTO LOSS & \\
\hline & TONS Kq'S DWN AVG MIN U PO & \\
\hline & & \\
\hline & $36.77 \ldots \quad 19,49-7.20 \quad 103-97-89-201$ & \\
\hline $\begin{array}{l}\text { FEB } \\
\text { MAN } \ldots \ldots\end{array}$ & $181.60 \quad 3359^{*} j 9.6 \quad 92 \quad 75 \quad 33-79$ & \\
\hline $\begin{array}{l}\text { WAR...... } \\
\text { APR....... }\end{array}$ & $245.40 \quad 4797^{*} 8.1-8276 \quad 46-82$ & \\
\hline $\begin{array}{l}\text { APR. } \\
\text { MLAY. }\end{array}$ & 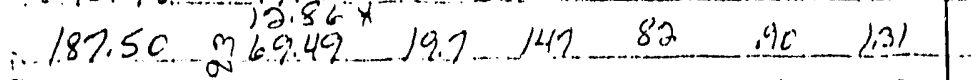 & \\
\hline $\begin{array}{l}\text { MLAY } \\
\text { JUNEE }\end{array}$ & $\because 256.17 \quad \begin{array}{lllllll}0 / 60.53 & 2.0 & 123 & 106 & .43 & .87\end{array}$ & $\cdots$ \\
\hline$\frac{J U N E}{F-5 S}$ & $\because 146.83 \quad 503.06 \quad 41.7$ 10c 86 .969 196 & \\
\hline$F y=55$ & 194282744242 & \\
\hline J'LY & 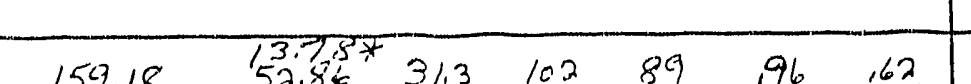 & \\
\hline Aus & 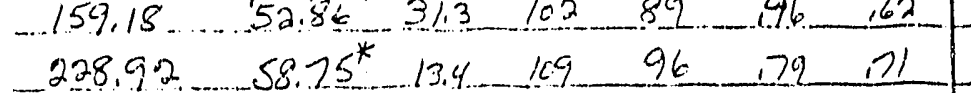 & \\
\hline SEP & $310.14 \quad 76.93^{*} 5 . \quad 100 \quad 85 \quad 43 \quad 76$ & \\
\hline CCT & $283.06 \cdot 56-77^{*} \quad 142 \quad 100 \quad 87 \quad 43 \quad 88$ & \\
\hline Nor & $236.90 \quad 9113.27 * 16.157-96 \quad .63 \quad 92$ & \\
\hline DEC & 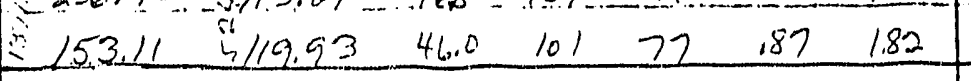 & \\
\hline$c y^{\prime} s 5$ & 2425.58971 .35 & \\
\hline & $\left(\begin{array}{l}638.63 \\
333.7 .25\end{array}\right) 245110 \min _{5} 62 \quad 1.02$ & \\
\hline 1956 & & \\
\hline SAN & $-115.46: 19.01^{*} 522-112 \quad 106-81 \quad 1.12$ & \\
\hline$F E B$ & $\begin{array}{lllllllll} & 246.94^{\prime} \quad 59.92^{*} \quad 16.8 & 114 & 103 & 08 & 43\end{array}$ & \\
\hline$M A R$ & 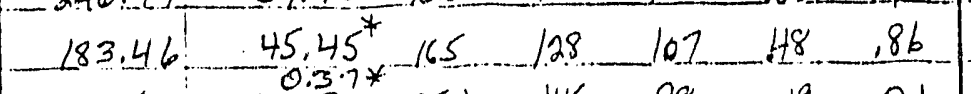 & \\
\hline APR:- & $200.60 \quad 13630^{*} \quad 35.1 \quad 145 \quad 98 \quad 48 \quad 121$ & \\
\hline MAY & $21423-134,24 \quad 270 \quad 109 \quad 97 \quad 25 \quad 175$ & \\
\hline JUNE & 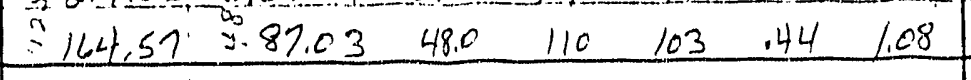 & \\
\hline EY 56 & 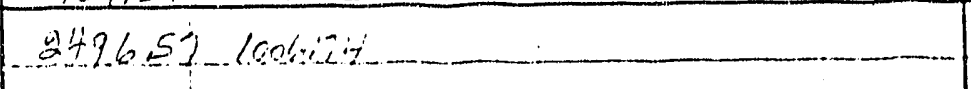 & \\
\hline & & \\
\hline$J u L Y$ & $5983, \quad 38.36 \quad 780 \quad 124 \quad 107 \quad .49 \quad 139$ & \\
\hline$A \cup G$ & $-10495 \quad 76.05 \quad 460 \quad 134 \quad 91 \quad 70 \quad 216$ & \\
\hline SEP & $241,30 \quad 57.26^{*} 17.5 \quad 148 \quad 121 \quad, 67 \quad, 56$ & \\
\hline $\mathbb{C} T$ & 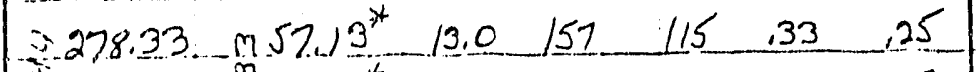 & $\vdots$ \\
\hline Nor & 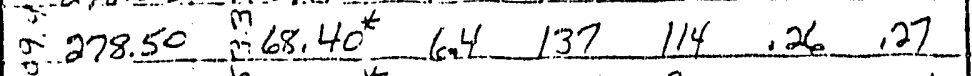 & 1 \\
\hline DEC. & $247.03 \quad 0.56 .13^{*} 17.8 \quad 114 \quad 90 \quad .23 \quad .26$ & \\
\hline$c y^{\prime} 56$ & $2335.20 \quad 835,65$ & \\
\hline & $-\left(\begin{array}{l}491,98 \\
36367 *\end{array}\right)^{3 / 2} \quad 90,36-178$ & \\
\hline & $* \tan -a / T$ & \\
\hline
\end{tabular}




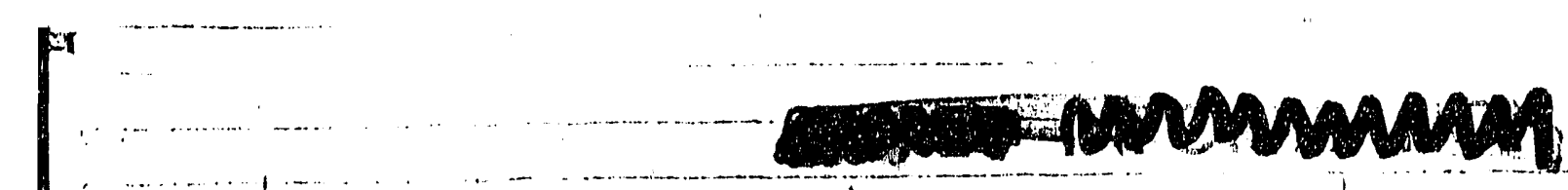

\begin{tabular}{|c|c|c|}
\hline \multirow{2}{*}{$\ldots \ldots+\cdots$} & $R E D O X$ & \\
\hline & $T C N J$ & \\
\hline & $K g^{\prime} s$ & \\
\hline \multicolumn{3}{|l|}{1057} \\
\hline & 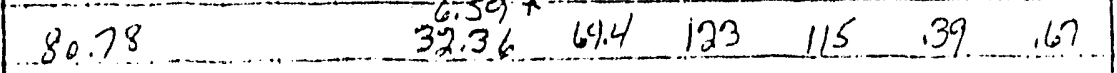 & \multirow{3}{*}{ 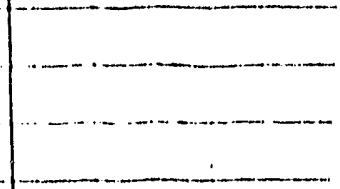 } \\
\hline$F E B$ & $72.8-58.6-120 \quad 109-17 \quad 43$ & \\
\hline$M A R$. & $26.00 \quad 640-128-107_{-1}-23-.87$ & \\
\hline$A P R$ & $.96 .23 \quad 41.4 \quad 119-105.40 \quad .83$ & \multirow{3}{*}{$\begin{array}{ll} \\
\ldots\end{array} \ldots$} \\
\hline MAY. & $\quad 599,00 \quad 39,2 \quad 121 \quad 97 \quad .10 \quad 39$ & \\
\hline JUNE & $\begin{array}{lllllll}100.160 & 59.45 & 67.8 & 174 & 107 & 18 & 16.9 \\
\end{array}$ & \\
\hline$E y-57$ & 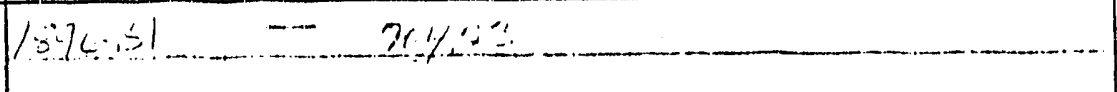 & \\
\hline SULY & \multicolumn{2}{|c|}{$171.79 \quad 67.59 \quad 338 \quad 118 \quad 108 \quad .23 \quad .57$} \\
\hline$A \cup G$ & \multicolumn{2}{|c|}{$153.17 \quad 73.40-33.413-104.09 \quad .39$} \\
\hline SEP & \multicolumn{2}{|c|}{219.46} \\
\hline $0 \subset T$ & \multicolumn{2}{|c|}{$181.641, \quad 80.92 .357 \ldots+110 \quad 96 \quad 124 \quad 85$} \\
\hline Nov & 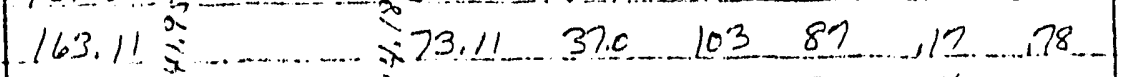 & \multirow[t]{2}{*}{ - } \\
\hline DEC & $253.78=\quad 5111.49 \quad 18.4 \quad 100 \quad 90 \quad 16 \quad 167$ & \\
\hline sy's? & \multicolumn{2}{|l|}{$1888.5 y-865.08$} \\
\hline & \multicolumn{2}{|c|}{$(858.49) 423 \quad 116 \quad 87, .19,62$} \\
\hline 1958 & \multicolumn{2}{|c|}{$(6.59 x)$} \\
\hline JA.N. & \multicolumn{2}{|c|}{$50.77 \quad 2 \% 10 \quad 71.4 \quad 104 \quad 89 \quad .75 \quad .68$} \\
\hline$F E B$ & \multicolumn{2}{|c|}{$131.92-65.92 \quad 44.390 \cdots 81 \quad 22 \quad .58$} \\
\hline MAR & \multicolumn{2}{|c|}{$104.39-55.0046092 .85 .27 \quad 39$} \\
\hline APR & \multicolumn{2}{|c|}{ 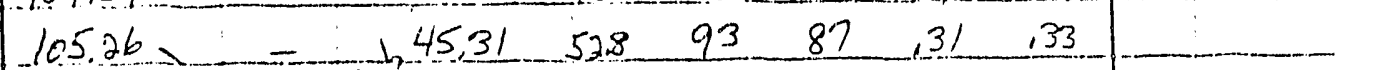 } \\
\hline MAY & \multicolumn{2}{|l|}{$160.780 .62 .4849 .59584,24 \quad 44$} \\
\hline JUNE & \multicolumn{2}{|l|}{ 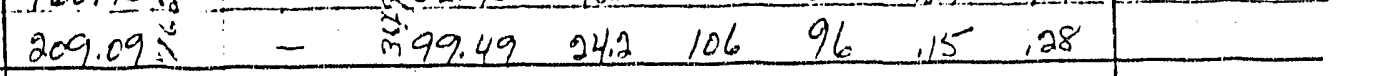 } \\
\hline EY' 58 & \multicolumn{2}{|c|}{$100416 \quad 8 \quad 8650$} \\
\hline & \multicolumn{2}{|c|}{ 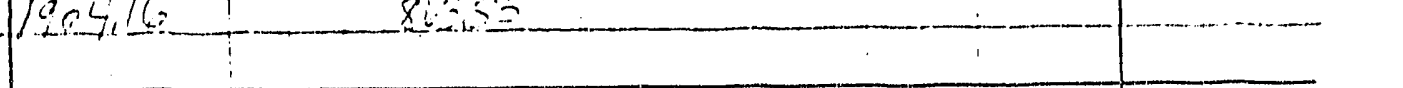 } \\
\hline JuLy & \\
\hline$A \cup G$. & \\
\hline SEP & \multicolumn{2}{|c|}{$14933-8463460 \quad 10994,15,51$} \\
\hline $\begin{array}{l}\text { OCT } \\
\text { NOV }\end{array}$ & \\
\hline NOV & \\
\hline$\frac{D E C}{C C Y ' s 8}$ & $\frac{0}{1312.94^{(2)}: 65.28}$ & \\
\hline & & \\
\hline & & \\
\hline
\end{tabular}
(5) A coun iability ReporT was $-5.46 T^{T} \mathrm{~T}$ Normal and $22.86 \mathrm{gg}$ Pu total

(7) Spe notes (5) and (6)

(i) Sep rote (6)

$$
-23-
$$


J7N $\quad 12.58 \quad 71.72 \quad 49.61 \quad 32.6 \quad 166 \quad 124 \quad 19 \quad, 50$ HEB - - $\quad 4773 \quad 2474 \quad 626 \quad 138 \quad 124 \quad 111 \quad 35$ MAR = - $54251934.95 \quad 728 \quad 150 \quad 110 \quad .25 .34$ APR $\therefore-61.09 m^{3} 4359-456145 \quad 122.11 .41$ MAY - $\quad 26.35 \quad 558 \quad 789 \quad 166 \quad 140,10 \quad 445$ Juke $115.13 \quad 36.98 \quad 72.06 \quad 25.0 \quad 136,110.13 \quad, 32$ $1560 \quad 187.71 \quad 64976 \quad 501.43$

\begin{tabular}{|c|c|c|}
\hline Suly & 34.78 & $589.173 \quad 103 \quad .15$ \\
\hline$A \cup G$ & $=21.51$ & 82.0130 \\
\hline EE & $=$ ij 24.44 & 62.0 .260 .237 \\
\hline CLT & $69.35 \div 52.96$ & 4450268239 \\
\hline & 51.43 & 32.5258 \\
\hline $\operatorname{CES}$ & 31.99 & $45,8 \quad 241$ \\
\hline$y^{\prime}$ & -437.04 & \\
\hline
\end{tabular}

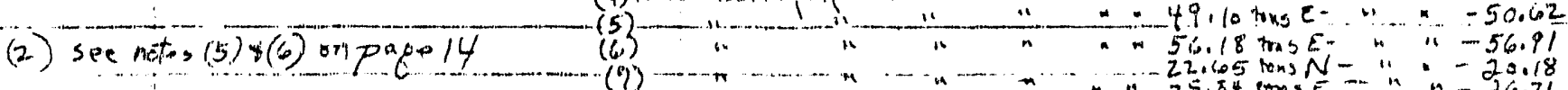

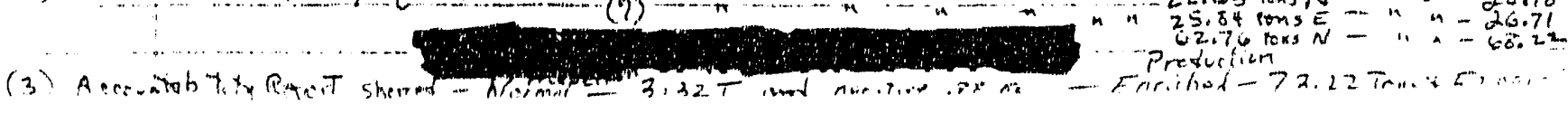

(8) Aciauntability Peport ihowed Preduetion to

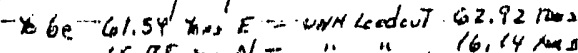

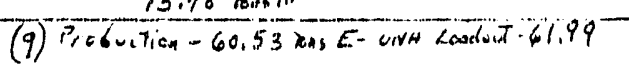




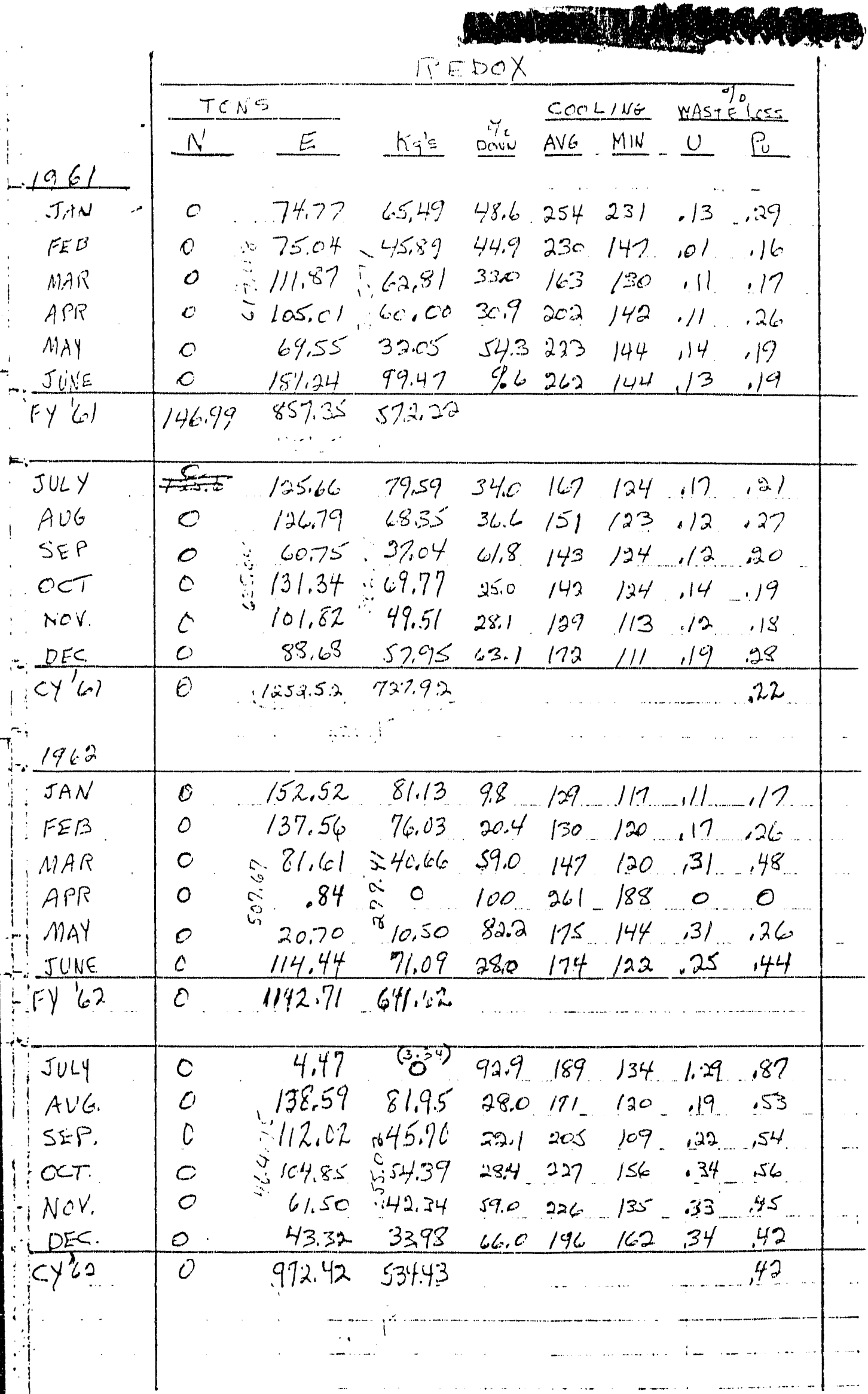




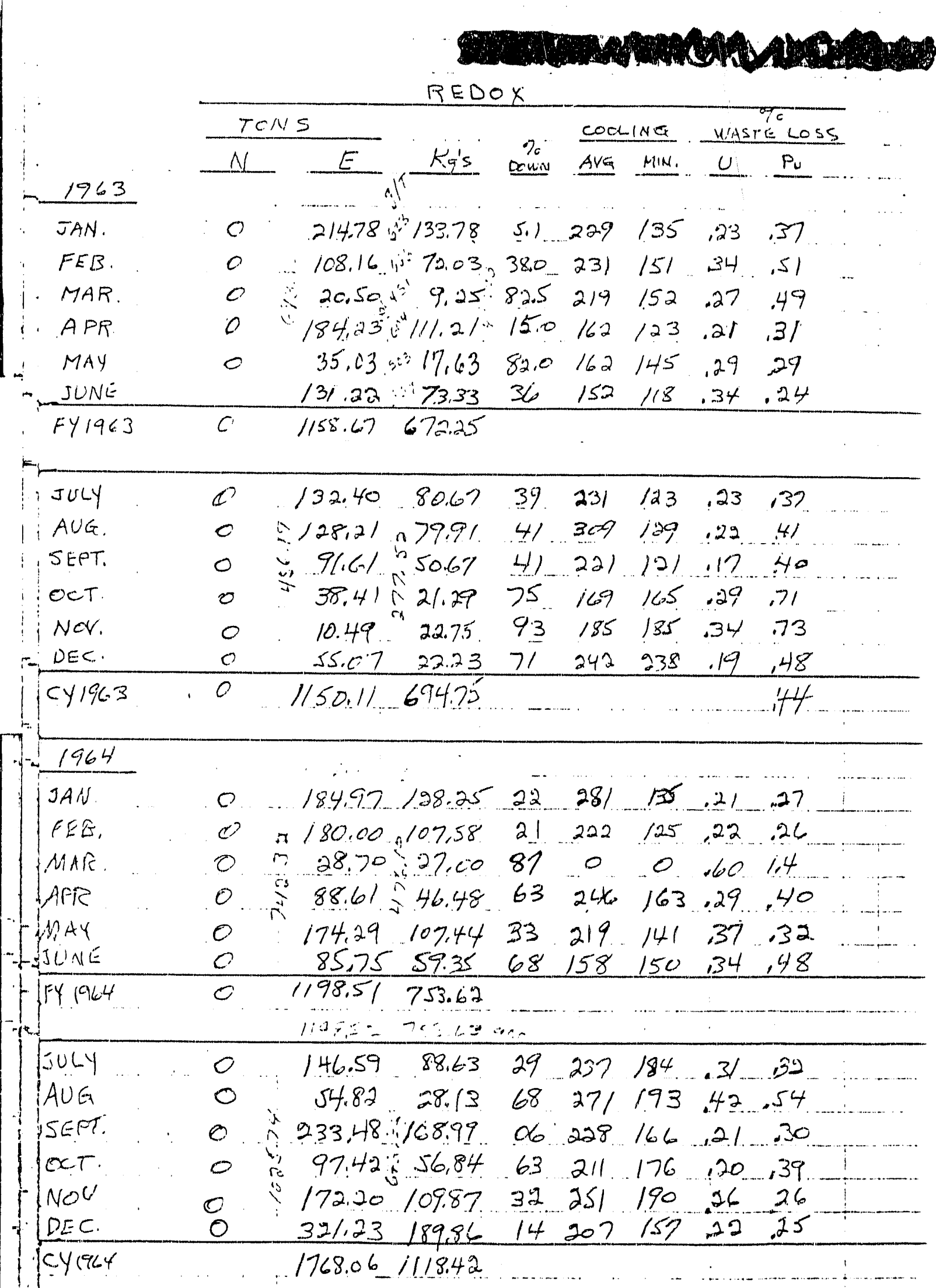




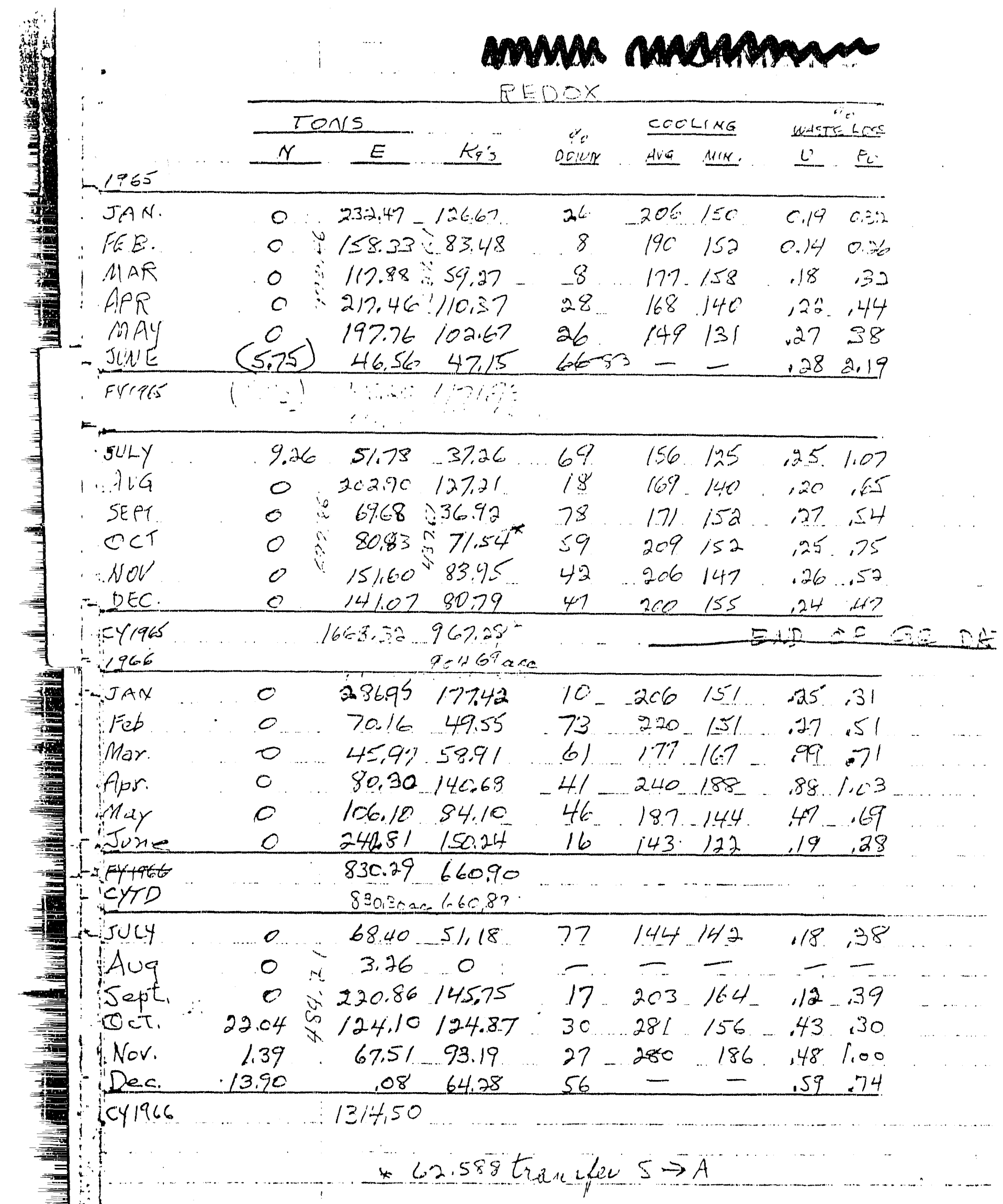



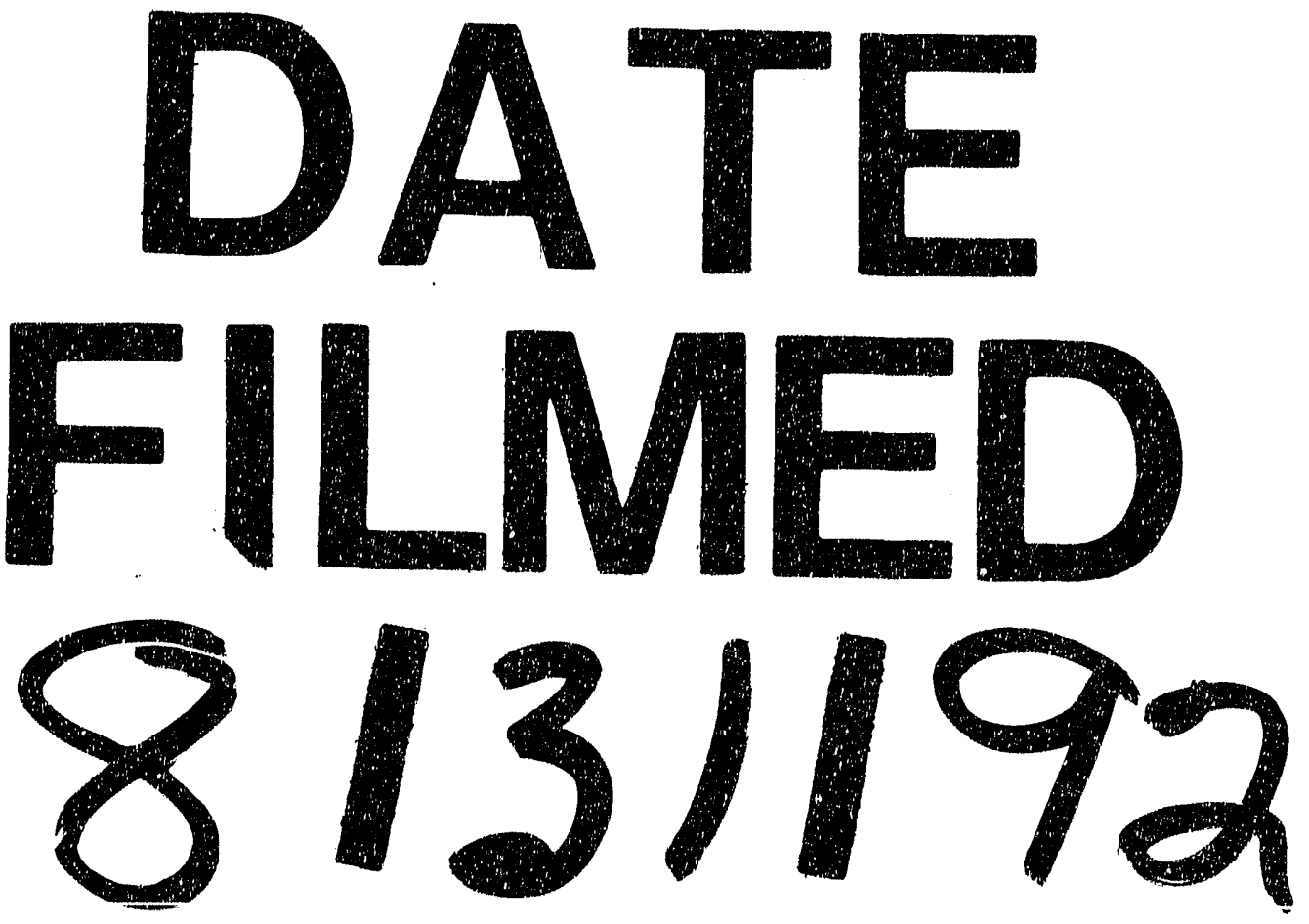
